\title{
Non-representable relation algebras from vector spaces
}

\author{
Ian Hodkinson* \\ Department of Computing, Imperial College London, \\ London SW7 2AZ, UK \\ i.hodkinson@imperial.ac.uk, www.doc.ic.ac.uk/ imh/
}

\begin{abstract}
Extending a construction of Andréka, Givant, and Németi (2019), we construct some finite vector spaces and use them to build finite non-representable relation algebras. They are simple, measurable, and persistently finite, and they validate arbitrary finite sets of equations that are valid in the variety RRA of representable relation algebras. It follows that there is no finitely axiomatisable class of relation algebras that contains RRA and validates every equation that is both valid in RRA and preserved by completions of relation algebras. Consequently, the variety generated by the completions of representable relation algebras is not finitely axiomatisable. This answers a question of Maddux (2018).
\end{abstract}

AMS 2020 classification $\quad 03 \mathrm{G} 15 ; 03 \mathrm{C} 05,15 \mathrm{~A} 03$

Keywords Non-representable simple measurable persistently finite relation algebras; completions of relation algebras; non-finitely axiomatisable varieties of relation algebras.

\section{Introduction}

This paper lies in the field of algebraic logic. It is concerned, at least on the face of it, with certain varieties of relation algebras lying between the variety RRA of representable relation algebras and the variety RA of all relation algebras.

The main one is the variety generated by the completions of algebras in RRA. It is an almost brand new variety, introduced by Maddux in [24], in which he denoted it by $V$. Every relation algebra is a subalgebra of its completion, which is a relation algebra, and it follows that RRA $\subseteq V \subseteq \mathrm{RA}$. It was shown in [14] that RRA is not closed under completions, so RRA $\subsetneq V$. In [24, problem 1.1], Maddux asked six questions about $V$ :

*This paper is dedicated to Rob Goldblatt on the occasion of his retirement. Thanks very much to Ed Mares for inviting this contribution and managing the process.

Australasian Journal of Logic (17:2) 2020, Article no. 1 
1. Is $V=\mathrm{RA}$ ?

2. Is $V$ finitely axiomatizable?

3. Is $V$ closed under canonical extensions?

4. Is $V$ closed under completions?

5. Is membership in $V$ decidable for finite algebras?

6. Does $V$ contain any algebras that are not weakly representable (as defined in [17])?

Already in his paper, Maddux proposed a way to answer the first question negatively. He suggested that finite (simple non-representable) relation algebras with no proper simple extensions were candidates for relation algebras that are not in $V$. It may seem surprising that such algebras could exist, but examples have been known since Frias and Maddux's paper [4].

Maddux's suggestion was soon taken up by Andréka and Németi, who showed in [2] that no non-representable simple persistently finite relation algebra can be in $V$. A relation algebra is persistently finite if it is finite and every simple relation algebra extending it is finite. Obviously, a finite relation algebra with no proper simple extensions is persistently finite, so Andréka and Németi's result validates and generalises Maddux's suggested approach.

Several kinds of non-representable simple persistently finite relation algebras are now known. As well as the examples from [4] already mentioned, the finite simple nonrepresentable 'coset relation algebras' constructed by Andréka, Givant, and Németi in [3] also pass muster, since they were shown to be persistently finite in [2, theorem 3.1] and the remarks following it. There are infinitely many algebras of both kinds, and each of them witnesses that $V \neq \mathrm{RA}$. Indeed, [2] goes on to show that there are continuum-many varieties between $V$ and RA. So the answer to Maddux's first question is resoundingly negative.

On seeing these relation algebras, one wonders if they could also be used to answer Maddux's second question, on whether $V$ is finitely axiomatisable. This could be done if they are arbitrarily representable in the sense that while being non-representable, there are algebras among them validating any desired finite subset of the equations defining RRA. Unfortunately, the examples from [4] all violate a particular equation '(L)' [4, theorem 7.1] known to be valid in RRA, and it appears that the examples from [3] do too.

In this paper, we extend the construction in [3]. We exhibit non-representable simple persistently finite relation algebras that are indeed arbitrarily representable. We know from [2] that they are not in $V$, but a nonprincipal ultraproduct of them will be in RRA and hence in $V$, from which we can deduce by Łos's theorem that $V$ is not finitely axiomatisable (theorem 4.10). So Maddux's second question also receives a negative answer.

In fact, we will take a slightly more general tack. Let $\mathcal{C}(\mathrm{RRA})$ be the variety defined by all equations that are valid in RRA and preserved by completions of relation algebras. Then RRA $\subsetneq V \subseteq \mathcal{C}(\mathrm{RRA}) \subseteq \mathrm{RA}$. Extending [2, theorem 4.3(iv)], we will show (in corollary 4.8) that no non-representable simple persistently finite relation algebra can lie in

Australasian Journal of Logic (17:2) 2020, Article no. 1 
$\mathcal{C}(\mathrm{RRA})$, yielding of course that $\mathcal{C}(\mathrm{RRA}) \subsetneq \mathrm{RA}$. We then conclude by the same ultraproduct argument, using the same algebras, that no variety lying between RRA and $\mathcal{C}$ (RRA) is finitely axiomatisable (theorem 4.9). This includes $V$ as well as $\mathcal{C}($ RRA) (and RRA).

These results are our main retail items, but our means of production may also be of interest. The simple persistently finite non-representable relation algebras constructed in [3] were in fact coset relation algebras. These form a sophisticated generalisation of group relation algebras, and are related to measurable relation algebras (see, e.g., [6]). They have been studied by Andréka, Givant and Németi in a long series of papers including [6]. References can be found in [3], together with a helpful introduction to the subject. For each finite abelian group $\mathfrak{F}$, [3] constructed a finite non-representable coset relation algebra from the group $\mathfrak{F}^{3}$. When $\mathfrak{F}$ is the additive group of a finite field of characteristic 2 , this is the ground case of an infinite sequence of relation algebras based on the additive groups of vector spaces constructed in $\S \S 2-3$ of this paper. They are non-representable simple persistently finite relation algebras, and they are arbitrarily representable.

In summary, we hope that the paper may be of use to those interested in:

- completions of relation algebras,

- non-finite axiomatisability of varieties of relation algebras,

- non-representable but arbitrarily representable simple persistently finite relation algebras,

- coset relation algebras (or measurable relation algebras),

- finite-dimensional vector spaces over finite fields.

We repeat that the work in this article is based on [3], and owes a great debt to it.

\section{$2 \quad$ Vector spaces}

Fix, for the rest of the paper, a finite field $\mathcal{F}$ of characteristic $2-$ so $\lambda+\lambda=0$ for every $\lambda$ in $\mathcal{F}$. The simplest example is of course $\mathbb{Z}_{2}$, the quotient of the integers $\mathbb{Z}$ by the even integers $2 \mathbb{Z}$. We are going to define and study some vector spaces over $\mathcal{F}$. In the end, we will be interested only in their abelian group structure, but using vector spaces gives us access to useful devices such as bases, dimension, and scalar (or inner) product. Finiteness of $\mathcal{F}$ is actually not needed at all in this section, but it is needed later to construct finite relation algebras from our vector spaces. Some, but perhaps not all, of what we do generalises to fields of other characteristics, and we will discuss this very briefly in the conclusion.

We use some standard notation. For a set $S$ and non-negative integers $k, n$, we write $S^{[k]}$ for the set $\{T \subseteq S:|T|=k\}$ of $k$-element subsets of $S$, and $\left(\begin{array}{l}n \\ k\end{array}\right)$ for $\left|n^{[k]}\right|$, where $n=\{0,1, \ldots, n-1\}$ as usual. For sets $S, T$, we write ${ }^{S} T$ for the set of maps $f: S \rightarrow T$. 


\subsection{The vector space $V_{n}^{(d)}$}

We now come to one of the main definitions of the paper.

DEFINITION 2.1 Let $d \geq 1$ and $n \geq 0$ be integers. We define the following items.

1. Let $K_{n}^{(d)}$ be a set of cardinality $n+d-1$. It is empty iff $n=0$ and $d=1$. This case is rather degenerate and not very useful, but we will keep an eye on it out of courtesy to the reader.

2. Let $E_{n}^{(d)}=\left(K_{n}^{(d)}\right)^{[d]}$. Thus, $\left(K_{n}^{(d)}, E_{n}^{(d)}\right)$ is the complete $d$-dimensional hypergraph over the set $K_{n}^{(d)}$ of nodes, with hyperedges of cardinality $d$. We will refer to these hyperedges (elements of $E_{n}^{(d)}$ ) simply as edges.

3. Let $V_{n}^{(d)}$ be the set ${ }^{(d)} \mathcal{F}$ of all maps from $E_{n}^{(d)}$ to $\mathcal{F}$. We regard $V_{n}^{(d)}$ as a vector space over $\mathcal{F}$ in the usual way by putting $(v+w)(e)=v(e)+w(e)$ and $(\lambda v)(e)=\lambda \cdot v(e)$, for $v, w \in V_{n}^{(d)}, \lambda \in \mathcal{F}$, and $e \in E_{n}^{(d)}$.

Where $d, n$ are fixed, we will drop the indices ${ }_{n}^{(d)}$ and write simply $K, E, V$ for the items defined above. Do not confuse this $V$ with Maddux's variety mentioned in the introduction. We use $a, b, p, q, r, x, y, z, \ldots$ to denote elements of $K, e, e^{\prime}$ to denote elements of $E$, and $v, u, w, g, h, k, \ldots$ to denote elements of (vectors in) $V$.

It is clear that the dimension $\operatorname{dim} V_{n}^{(d)}$ of the vector space $V_{n}^{(d)}$ is equal to $\left|E_{n}^{(d)}\right|$, which is $\left|\left(K_{n}^{(d)}\right)^{[d]}\right|=\left(\begin{array}{c}n+d-1 \\ d\end{array}\right)$. When $n \geq 1$, this is equal to $\left(\begin{array}{c}n+d-1 \\ n-1\end{array}\right)$. So that we can see what we are up against, table 1 lists a few values of this dimension.

\begin{tabular}{l|ccccccc}
$d \backslash n$ & 0 & 1 & 2 & 3 & 4 & 5 & $n$ \\
\hline 1 & 0 & 1 & 2 & 3 & 4 & 5 & $\left(\begin{array}{c}n \\
1\end{array}\right)$ \\
2 & 0 & 1 & 3 & 6 & 10 & 15 & $\left(\begin{array}{c}n+1 \\
2\end{array}\right)$ \\
3 & 0 & 1 & 4 & 10 & 20 & 35 & $\left(\begin{array}{c}n+2 \\
3\end{array}\right)$ \\
$d$ & 0 & 1 & $d+1$ & $\left(\begin{array}{c}d+2 \\
d\end{array}\right)$ & $\left(\begin{array}{c}d+3 \\
d\end{array}\right)$ & $\left(\begin{array}{c}d+4 \\
d\end{array}\right)$ & $\left(\begin{array}{c}n+d-1 \\
d\end{array}\right)$
\end{tabular}

Table 1: Some values of $\operatorname{dim} V_{n}^{(d)}$

When $n=0$, we have $\left|K_{0}^{(d)}\right|=d-1$, so $E_{0}^{(d)}=\emptyset$. Then $V_{0}^{(d)}={ }^{\emptyset} \mathcal{F}=\{\emptyset\}$ since $\emptyset$ is the unique function from $\emptyset$ to $\mathcal{F}$. This is the degenerate 0 -dimensional vector space over $\mathcal{F}$.

Later, $n$ will mainly be 5 , but allowing arbitrary $n$ has its uses, takes no more effort to handle, and in fact makes things clearer.

\subsection{Some useful notation}

For the rest of $\S 2$, we fix integers $d \geq 1$ and $n \geq 0$. Unadorned $K, E, V$ will denote $K_{n}^{(d)}$, $E_{n}^{(d)}$, and $V_{n}^{(d)}$, respectively. 


\section{DEFINITION 2.2}

1. For $S \subseteq E$, we write $\chi_{S}: E \rightarrow \mathcal{F}$ for the characteristic function of $S$ :

$$
\chi_{S}(e)= \begin{cases}1, & \text { if } e \in S, \\ 0 & \text { otherwise }\end{cases}
$$

for $e \in E$. Here, 0 and 1 are taken in the field $\mathcal{F}$. So $\chi_{S} \in V$.

2. Plainly, $\mathcal{B}=\left\{\chi_{\{e\}}: e \in E\right\}$ is a basis of $V$, and we call it the standard basis.

3. For $W \subseteq V$, we write $\langle W\rangle$ for the subspace of $V$ spanned (generated) by $W$.

As examples, $v=\sum_{e \in E} v(e) \cdot \chi_{\{e\}} \in\langle\mathcal{B}\rangle$ for $v \in V$, and $\chi_{S}=\sum_{e \in S} \chi_{\{e\}}$ for $S \subseteq E$. The subspace $\langle W\rangle$ is the set of linear combinations of elements of $W$.

We now pick out some useful sets of edges.

DEFINITION 2.3 For $X \subseteq K$, we define

- $X \uparrow=\{e \in E: X \subseteq e\}$,

- $X \downarrow=\{e \in E: e \subseteq X\}$.

The latter is of course $X^{[d]}$, but the notation $\downarrow$ is more to the point here. For $a \in K$, we write $a \uparrow$ as short for $\{a\} \uparrow$.

\subsection{The subspaces $L_{a}$ of $V(a \in K)$}

Now we define the first of two kinds of subspace of $V$.

\section{DEFINITION 2.4}

1. For $v \in V$, we write $\operatorname{supp}(v)=\{e \in E: v(e) \neq 0\}$ - the support of $v$.

2. For $a \in K$, we define $L_{a}=\{v \in V: \operatorname{supp}(v) \subseteq a \uparrow\}$, and $\mathcal{B}_{a}=\left\{\chi_{\{e\}}: e \in a \uparrow\right\}$.

Equivalently, $L_{a}=\{v \in V: \forall e \in E(v(e) \neq 0 \rightarrow a \in e)\}$. When $K$ is empty, no $L_{a}$ are defined.

LEMMA 2.5 Let $a \in K$. Then $L_{a}=\left\langle\mathcal{B}_{a}\right\rangle$.

Proof. We show first that $L_{a}$ is a subspace of $V$. If $v, w \in V$ and $\lambda \in \mathcal{F}$ then $\operatorname{supp}(v+w) \subseteq$ $\operatorname{supp}(v) \cup \operatorname{supp}(w)$ and $\operatorname{supp}(\lambda v) \subseteq \operatorname{supp}(v)$. It follows by definition of $L_{a}$ that if $v, w \in L_{a}$ and $\lambda \in \mathcal{F}$ then $v+w, \lambda v \in L_{a}$, as required.

Plainly, $\mathcal{B}_{a} \subseteq L_{a}$, so $\left\langle\mathcal{B}_{a}\right\rangle \subseteq L_{a}$ since $L_{a}$ is a subspace of $V$. Conversely, if $v \in L_{a}$, then since $\operatorname{supp}(v) \subseteq a \uparrow$, so $v(e)=0$ whenever $e \in E \backslash a \uparrow$, we see that

$$
v=\sum_{e \in E} v(e) \chi_{\{e\}}=\sum_{e \in a \uparrow} v(e) \chi_{\{e\}} \in\left\langle\mathcal{B}_{a}\right\rangle .
$$

So $\left\langle\mathcal{B}_{a}\right\rangle=L_{a}$, proving the lemma.

Australasian Journal of Logic (17:2) 2020, Article no. 1 
Since $\mathcal{B}_{a}$ is a subset of the standard basis $\mathcal{B}$ of $V$, it is linearly independent. Hence, it is actually a basis of $L_{a}$, giving

$$
\operatorname{dim} L_{a}=\left|\mathcal{B}_{a}\right|=|a \uparrow|=\left|(K \backslash\{a\})^{[d-1]}\right|=\left(\begin{array}{c}
n+d-2 \\
d-1
\end{array}\right)\left(=\operatorname{dim} V_{n}^{(d-1)} \text { if } d>1\right) .
$$

To see the third equality here, observe that $e \mapsto e \backslash\{a\}$ is a bijection from $a \uparrow$ to $(K \backslash\{a\})^{[d-1]}$.

\subsection{The diagonal subspace $L_{\Delta}$}

One more 'diagonal' subspace of $V$ is defined as follows. We take $\Delta$ to be a special symbol not in any set $K_{n}^{(d)}$.

DEFINITION 2.6 Let $L_{\Delta}$ be the subspace of $V$ spanned by $\mathcal{X} \stackrel{\text { def }}{=}\left\{\chi_{(X \uparrow)}: X \in K^{[d-1]}\right\}$.

The definition of $L_{\Delta}$ is very different from that of the $L_{a}(a \in K)$, but it is really part of the same family. We will not need to show that every permutation of $\left\{L_{x}: x \in K \cup\{\Delta\}\right\}$ is induced by an automorphism of $V$, but it is true, and a related result is proved in [3, lemma 3.1]. It follows that $L_{\Delta}$ has the same dimension as the $L_{a}(a \in K)$.

If $K$ is empty, so $n=0$ and $d=1$, then $\mathcal{X}=\left\{\chi_{(X \uparrow)}: X \in \emptyset^{[0]}\right\}=\left\{\chi_{(\emptyset \uparrow)}\right\}=\left\{\chi_{E}\right\}$. Here, $E=\emptyset^{[1]}=\emptyset$ too, so $\mathcal{X}=\{\emptyset\}$. But $V={ }^{E} \mathcal{F}=\{\emptyset\}$ as well. So $L_{\Delta}=\langle\mathcal{X}\rangle=V$. We see that $L_{\Delta}$ is defined in this case, but both it and $V$ are degenerate.

\subsection{Sums of the subspaces}

We now examine combinations of these subspaces. Our findings will be useful in $\S 3$.

DEFINITION 2.7 For $S \subseteq K \cup\{\Delta\}$, we define $L_{S}=\sum_{x \in S} L_{x} \subseteq V$.

Each $L_{x}(x \in K \cup\{\Delta\})$ is a subspace of $V$, so $L_{S}$ is also a subspace of $V$. Several times we will use the obvious fact that if $S \subseteq S^{\prime} \subseteq K \cup\{\Delta\}$ then $L_{S} \subseteq L_{S^{\prime}}$.

In the case where $S \subseteq K$, it can be checked that for each $v \in V$ we have $v \in L_{S}$ iff $v(e)=0$ for all $e \in E$ disjoint from $S$. We will not need this. However, we will need (in propositions 3.13 and 3.15) a similar characterisation of when an arbitrary $v \in V$ is in $L_{S \cup\{\Delta\}}$, and we give one in proposition 2.12. The two lemmas coming up are needed to prove this proposition. The first one uses that $\mathcal{F}$ has characteristic 2 .

As usual, for vectors $v, w \in V$, we define their scalar product $v \cdot w=\sum_{e \in E}(v(e) \cdot w(e)) \in$ $\mathcal{F}$, where the $\sum$ and $\cdot$ on the right are taken in the field $\mathcal{F}$. As examples, for $v \in V$, if $e \in E$ then $v \cdot \chi_{\{e\}}=v(e)$, and if $T \subseteq E$ then $v \cdot \chi_{T}=\sum_{e \in T} v(e)$. Scalar product is linear in both arguments.

Frequently we will write $X$ for a subset of $K$ of size $d-1$, and $Y$ for one of size $d+1$.

LEMMA 2.8 Let $S \subseteq K$ and let $Y \in K^{[d+1]}$ be disjoint from $S$. Then $v \cdot \chi_{Y \downarrow}=0$ for every $v \in L_{S \cup\{\Delta\}}$. 
Proof. By lemma 2.5 and definitions 2.6 and 2.7, $L_{S \cup\{\Delta\}}=\left\langle\bigcup_{s \in S} \mathcal{B}_{s} \cup \mathcal{X}\right\rangle$. So by linearity of scalar product, it is sufficient to prove that $v \cdot \chi_{Y \downarrow}=0$ for each $v \in \bigcup_{s \in S} \mathcal{B}_{s} \cup \mathcal{X}$.

If $v=\chi_{\{e\}} \in \mathcal{B}_{s}$ for some $s \in S$, then $e \notin Y \downarrow$ since $s \in e \backslash Y$, and so $v \cdot \chi_{Y \downarrow}=\chi_{Y \downarrow}(e)=0$ as required.

Suppose that $v=\chi_{X \uparrow} \in \mathcal{X}$. Then $|X|=d-1,|Y|=d+1$, and edges have size $d$, and it follows that

$$
|X \uparrow \cap Y \downarrow|= \begin{cases}2, & \text { if } X \subseteq Y, \\ 0, & \text { otherwise. }\end{cases}
$$

This value is always even. So $v \cdot \chi_{Y \downarrow}=\sum_{e \in X \uparrow \cap Y \downarrow} 1=0$, since $\mathcal{F}$ has characteristic 2 .

The following corollary will be used in definition 3.2 .

COROLLARY 2.9 Suppose that $S \subseteq K$ and $|S| \leq n-2$. Then $L_{S \cup\{\Delta\}} \subsetneq V$.

Proof. Since $|S| \leq n-2$, we have $|K \backslash S|=|K|-|S| \geq(n+d-1)-(n-2)=d+1$, so there exists $Y \in K^{[d+1]}$ disjoint from $S$. Take any edge $e \in Y \downarrow$. Then $\chi_{\{e\}} \cdot \chi_{Y \downarrow}=\chi_{Y \downarrow}(e)=1$. So $\chi_{\{e\}} \in V \backslash L_{S \cup\{\Delta\}}$ by lemma 2.8 .

DEFINITION 2.10 For $S \subseteq K$ and $a \in K \backslash S$, let $\mathcal{E}_{S}^{a}=\left\{\chi_{\{e\}}: e \in E\right.$, en $\left.(S \cup\{a\})=\emptyset\right\}$.

LEMMA 2.11 Let $S \subseteq K$ and $a \in K \backslash S$. Then $V=L_{S \cup\{\Delta\}}+\left\langle\mathcal{E}_{S}^{a}\right\rangle$.

Proof. We write $\mathcal{E}_{S}^{a}$ simply as $\mathcal{E}$. It is enough to show that the standard basis $\mathcal{B}$ of $V$ is contained in $L_{S \cup\{\Delta\}}+\langle\mathcal{E}\rangle$. So let $\chi_{\{e\}} \in \mathcal{B}$. If $e \cap S \neq \emptyset$, then there is $s \in e \cap S$, and so $\chi_{\{e\}} \in L_{s} \subseteq L_{S \cup\{\Delta\}}$. If $e \cap(S \cup\{a\})=\emptyset$, then $\chi_{\{e\}} \in \mathcal{E}$.

The remaining case is when $e$ is disjoint from $S$ and contains $a$. Let $X=e \backslash\{a\} \in K^{[d-1]}$. Now obviously,

$$
\begin{aligned}
X \uparrow & =\{X \cup\{b\}: b \in K \backslash X\} \\
& =\{e\} \cup\{X \cup\{s\}: s \in S\} \cup\{X \cup\{b\}: b \in K \backslash(S \cup\{a\})\}, \\
\text { so } \quad \chi_{X \uparrow} & =\sum_{e^{\prime} \in X \uparrow} \chi_{\left\{e^{\prime}\right\}}=\chi_{\{e\}}+\sum_{s \in S} \chi_{\{X \cup\{s\}\}}+\sum_{b \in K \backslash(S \cup\{a\})} \chi_{\{X \cup\{b\}\}} .
\end{aligned}
$$

Rearranging this (bearing in mind that $\mathcal{F}$ has characteristic 2) gives

$$
\chi_{\{e\}}=\underbrace{\chi_{X \uparrow}}_{L_{\Delta}}+\underbrace{\sum_{s \in S} \chi_{\{X \cup\{s\}\}}}_{L_{S}}+\underbrace{\sum_{b \in K \backslash(S \cup\{a\})} \chi_{\{X \cup\{b\}\}}}_{\langle\mathcal{E}\rangle} .
$$

Now $\chi_{X \uparrow} \in \mathcal{X} \subseteq L_{\Delta}$ by definition 2.6. For each $s \in S$ we have $\chi_{\{X \cup\{s\}\}} \in L_{s}$ by definition 2.4, since $X \cup\{s\} \in s \uparrow$. And for each $b \in K \backslash(S \cup\{a\})$ we have $\chi_{\{X \cup\{b\}\}} \in \mathcal{E}$ by definition 2.10. We conclude from (1) that $\chi_{\{e\}} \in L_{S \cup\{\Delta\}}+\langle\mathcal{E}\rangle$, as required.

As promised, we now characterise when a vector is in $L_{S \cup\{\Delta\}}$.

Australasian Journal of Logic (17:2) 2020, Article no. 1 
PROPOSITION 2.12 Let $S \subseteq K$. Then for each $v \in V$, the following are equivalent:

L1. $v \in L_{S \cup\{\Delta\}}$,

L2. $v \cdot \chi_{Y \downarrow}=0$ for every $Y \in K^{[d+1]}$ disjoint from $S$,

L3. $S=K$, or for some $a \in K \backslash S$ we have $v \cdot \chi_{Y \downarrow}=0$ for every $Y \in K^{[d+1]}$ disjoint from $S$ and containing a.

Proof. Fix any $v \in V$. By lemma 2.8, L1 of the proposition implies L2. If L2 holds, then L3 trivially follows as a special case. Now assume L3; we will prove L1 - that is, $v \in L_{S \cup\{\Delta\}}$.

There are two cases. If $S=K$, then already $L_{S}=V$ - trivially if $K=\emptyset$, and also if $K \neq \emptyset$ since each member $\chi_{\{e\}}$ of the standard basis $\mathcal{B}$ is in $L_{a}$ for every $a \in e$, and hence in $L_{S}$. So certainly, $v \in L_{S \cup\{\Delta\}}$.

The second case is when $S \neq K$. By L3, we can choose $a \in K \backslash S$ with $v \cdot \chi_{Y \downarrow}=0$ for every $Y \in K^{[d+1]}$ disjoint from $S$ and containing $a$. Let $\mathcal{E}=\mathcal{E}_{S}^{a}$ as in definition 2.10. By lemma 2.11,

$$
v=w+\sum_{\gamma \in \mathcal{E}} \lambda_{\gamma} \gamma
$$

for some $w \in L_{S \cup\{\Delta\}}$ and $\lambda_{\gamma} \in \mathcal{F}$. We show that each $\lambda_{\gamma}$ is zero, so that $v=w \in L_{S \cup\{\Delta\}}$.

So take any $\gamma=\chi_{\{e\}} \in \mathcal{E}$. So $e \in E$ is disjoint from $S \cup\{a\}$. Let $Y=e \cup\{a\}$. Then $Y \in K^{[d+1]}$, and $Y$ is disjoint from $S$ and contains $a$. We have the following:

- $w \cdot \chi_{Y \downarrow}=0$ by lemma 2.8 .

- $\gamma \cdot \chi_{Y \downarrow}=\chi_{Y \downarrow}(e)=1$ since $e \in Y \downarrow$.

- For every $\gamma^{\prime}=\chi_{\left\{e^{\prime}\right\}} \in \mathcal{E} \backslash\left\{\chi_{\{e\}}\right\}$, we have $e^{\prime} \nsubseteq e$ and $a \notin e^{\prime}$, so $e^{\prime} \nsubseteq e \cup\{a\}=Y$, giving $e^{\prime} \notin Y \downarrow$ and hence $\gamma^{\prime} \cdot \chi_{Y \downarrow}=\chi_{Y \downarrow}\left(e^{\prime}\right)=0$.

So by (2) and linearity of scalar product,

$$
v \cdot \chi_{Y \downarrow}=w \cdot \chi_{Y \downarrow}+\sum_{\gamma^{\prime} \in \mathcal{E}} \lambda_{\gamma^{\prime}}\left(\gamma^{\prime} \cdot \chi_{Y \downarrow}\right)=\lambda_{\gamma}\left(\gamma \cdot \chi_{Y \downarrow}\right)=\lambda_{\gamma}
$$

But $v \cdot \chi_{Y \downarrow}=0$ by choice of $a$, so $\lambda_{\gamma}=0$ as desired.

Taking $S=\emptyset$ in proposition 2.12 yields $L_{\Delta}=\left\{v \in V: v \cdot \chi_{Y \downarrow}=0\right.$ for every $\left.Y \in K^{[d+1]}\right\}$, and this is an alternative definition of $L_{\Delta}$.

We can also see that corollary 2.9 is optimal. For suppose that $S \subseteq K$ and $|S| \geq n-1$. Then $|K \backslash S| \leq d$, and so no $Y \in K^{[d+1]}$ is disjoint from $S$. Consequently, for every $v \in V$, L2 of proposition 2.12 holds vacuously, and hence L1 holds. We conclude that $L_{S \cup\{\Delta\}}=V$.

Australasian Journal of Logic (17:2) 2020, Article no. 1 


\section{Relation algebras from vector spaces}

We now fix $n=5$, but $d \geq 1$ remains arbitrary. Thus, $K, E, V$ denote $K_{5}^{(d)}, E_{5}^{(d)}$, and the vector space $V_{5}^{(d)}$, respectively. So $|K|=d+4$ and $\operatorname{dim} V=\left(\begin{array}{c}d+4 \\ d\end{array}\right)$, or equally, $\left(\begin{array}{c}d+4 \\ 4\end{array}\right)$. Since the field $\mathcal{F}$ is finite, so is $V$. We will still be using the internal structure of $V$, so the reader will need to bear in mind more of $\S 2$ than just the bare results proved there.

In this long section, we will define a finite relation algebra $\mathcal{A}^{(d)}$ over $V$, and prove some things about it: to wit, it has a $(d+3)$-dimensional cylindric basis but no $(d+4)$ dimensional relational basis, and it is simple, measurable, and persistently finite. These results may be of interest in their own right, irrespective of the applications they find in $\S 4$. We will recall the relevant relation algebra concepts as we go. Readers who would like an introduction to relation algebras are referred to the introduction of [24], which is worth reading in any case, and the survey and books $[22,23,13]$, but many other options exist in the literature.

\subsection{Non-associative, weakly associative, and relation algebras}

A non-associative algebra is an algebra of the form

$$
\mathcal{A}=\left(A,+,-, 0,1,1^{\prime},{ }^{\cup}, ;\right),
$$

where $(A,+,-, 0,1)$ is a boolean algebra, $1 '$ is a constant called identity, ' $`$ is a unary function called converse, ; is a binary function called composition, $a ; 1^{\prime}=1^{\prime} ; a=a$ holds for every $a \in A$, and the three conditions $a \cdot(b ; c)=0, b \cdot(a ; \breve{c})=0$, and $c \cdot(\breve{b} ; a)=0$ are equivalent for every $a, b, c \in A$. Here, as usual, $a \cdot b$ denotes $-(-a+-b)$. We define the usual partial ordering $\leq$ on $A$ by $a \leq b$ iff $a+b=b$. We say that $\mathcal{A}$ is a weakly associative algebra if $(a ; 1) ; 1=a ;(1 ; 1)$ for every $a \in A$ with $a \leq 1$, , and a relation algebra if the operation ; is associative. Obviously, every relation algebra is weakly associative.

Whilst these definitions are not equational, they can be replicated by equations, and so the classes of non-associative algebras and weakly associative algebras and the class RA of relation algebras are varieties — classes of algebras defined by equations [19, corollary 1.5].

Examples of relation algebras are algebras of the form

$$
\mathfrak{R e}(U)=\left(\wp(U \times U), \cup, \sim, \emptyset, U \times U, I d_{U},-^{-1}, \mid\right),
$$

where $U$ is a set, $I d_{U}=\{(u, u): u \in U\}$, and for each $a, b \subseteq U \times U, a^{-1}=\{(v, u):(u, v) \in$ $a\}$, and $a \mid b=\{(u, v):(u, w) \in a$ and $(w, v) \in b$ for some $w \in U\}$. So $\mathfrak{R e}(U)$ is the set of all binary relations on $U$, endowed with the indicated operations.

The class RRA of representable relation algebras is defined to be $\operatorname{SP}\{\mathfrak{R e}(U): U$ a set\}, where $\mathbf{P}$ and $\mathbf{S}$ denote closure under products and isomorphic copies of subalgebras, respectively. It is easy to see that RRA $\subseteq$ RA. We say that a relation algebra is representable if it is in RRA. Non-trivially, RRA is a non-finitely axiomatisable variety [25, 27].

We import some boolean algebra notions to non-associative algebras. Let $\mathcal{A}=(A,+,-$, $0,1,1,,, ;)$ be a non-associative algebra. An atom of $\mathcal{A}$ is a $\leq$-minimal non-zero element

Australasian Journal of Logic (17:2) 2020, Article no. 1 
of $A$, and $\mathcal{A}$ is said to be atomic if for every non-zero element $a \in A$, there is an atom $b$ of $\mathcal{A}$ with $b \leq a$. All finite non-associative algebras are atomic.

We say that $\mathcal{A}$ is complete if every set $S$ of elements of $A$ has a sum $\sum S$ in $\mathcal{A}$ - the least upper bound of $S$ with respect to $\leq$. By the De Morgan laws, it is of course equivalent to say that every $S \subseteq A$ has a product $\prod S$ - its greatest lower bound with respect to $\leq$. As examples, $\mathfrak{R e}(U)$ is of course always complete, and all finite non-associative algebras are complete.

\subsection{The algebra $\mathcal{A}^{(d)}$}

We now define a finite non-associative algebra $\mathcal{A}^{(d)}$ over $V$, which we will show later (in corollary 3.14) to be a relation algebra. The algebra $\mathcal{A}^{(d)}$ is what is known as the full complex algebra over a certain atom structure, but we will define it directly. It will help to bear in mind that $|K|=d+4 \geq 5$. We use the following convention frequently.

NOTATION 3.1 For $x_{1}, \ldots, x_{m} \in K \cup\{\Delta\}$, we write $L_{x_{1} \ldots x_{m}}$ as short for $L_{\left\{x_{1}, \ldots, x_{m}\right\}}$ (see definition 2.7). So, for example, $L_{x y}, L_{y x}, L_{x x y}, \ldots$, all denote $L_{\{x, y\}}$.

\section{DEFINITION 3.2}

1. Fix pairwise distinct elements $p, q, r \in K$.

2. Fix a vector $c_{S} \in V$ for each $S \subseteq K$ with $1 \leq|S| \leq 3$, such that:

- $c_{\{p, q, r\}} \notin L_{p q r \Delta}$. This is possible because $n=5$, so $|\{p, q, r\}| \leq n-2$, and hence $L_{p q r \Delta} \neq V$ by corollary 2.9 .

- if $S \neq\{p, q, r\}$ then $c_{S}=0$.

Extending notation 3.1, for $x, y, z \in K$ we write $c_{x y z}$ as short for $c_{\{x, y, z\}}$.

3. Let $\mathcal{S}$ be the set of all triples $\left(x, L_{x y \Delta}+v, y\right)$, where $x, y \in K$ and $v \in V$. As usual here, $L_{x y \Delta}+v$ is the coset $\left\{w+v: w \in L_{x y \Delta}\right\}$ of the subgroup $L_{x y \Delta}$ of the additive group of $V$. It is an element of the quotient space $V / L_{x y \Delta}$.

For $s=\left(x, L_{x y \Delta}+v, y\right) \in \mathcal{S}$, we write $\operatorname{start}(s)=x$ and end $(s)=y$. We can regard $s$ as 'going from $x$ to $y$ '.

For $x, y \in K$ and $v \in V$, we will write $(x, \widehat{v}, y)$ as shorthand for $\left(x, L_{x y \Delta}+v, y\right) \in \mathcal{S}$. So $\widehat{v}$ is $L_{x y \Delta}+v$, the $x$ and $y$ here being determined by context. Thus, the meaning of $\widehat{v}$ in $(x, \widehat{v}, y)$ involves all of $v, x, y$. Of course, $(x, \widehat{v}, y)=(x, \widehat{w}, y)$ iff $v+w \in L_{x y \Delta}$. So for each $s \in \mathcal{S}$ we have $s=(\operatorname{start}(s), \widehat{v}$, end $(s))$, for some (not unique) $v \in V$.

4. The domain of $\mathcal{A}^{(d)}$ is $\wp(\mathcal{S})$. 
5. The boolean operations $+,-, 0,1$ on $\mathcal{A}^{(d)}$ are defined as usual on power sets: + is union, - is unary complement, 0 is $\emptyset$, and 1 is $\mathcal{S}$. Under these operations, $\mathcal{A}^{(d)}$ is an atomic boolean algebra and its atoms are the singletons $\{s\}$ for $s \in \mathcal{S}$. We will usually identify the atom $\{s\}$ with the element $s \in \mathcal{S}$.

6. The identity 1' of $\mathcal{A}^{(d)}$ is the set $\{(x, \widehat{0}, x): x \in K\} \subseteq \mathcal{S}$. Here, $\widehat{0}$ is of course the coset $L_{x x \Delta}+0=L_{x \Delta}$ of $L_{x \Delta}$ - the zero or identity element of the quotient space $V / L_{x \Delta}$.

7. Converse is defined on atoms by $\left(x, L_{x y \Delta}+v, y\right)^{\triangleleft}=\left(y, L_{y x \Delta}+v, x\right)$. This is plainly well defined - independent of the choice of $v$ - because $L_{y x \Delta}=L_{x y \Delta}\left(=L_{\{x, y, \Delta\}}\right)$. In other words, $(x, \widehat{v}, y)^{\triangleleft}=(y, \widehat{v}, x)$. So we reverse the order of the entries and don't change the central term at all.

For arbitrary $a$ in $\mathcal{A}^{(d)}$, we then define $\breve{a}=\{\breve{s}: s \in a\}$.

8. Composition is defined by $a ; b=\{s \in \mathcal{S}: s \leq t ; u$ for some $t \in a, u \in b\}$ for $a, b \subseteq \mathcal{S}$, where the ternary relation ' $s \leq t ; u$ ' on $\mathcal{S}$ is defined as follows.

Let $s=(x, \widehat{k}, z), t=\left(x^{\prime}, \widehat{g}, y^{\prime}\right)$, and $u=\left(y^{\prime \prime}, \widehat{h}, z^{\prime \prime}\right)$ be arbitrary elements of $\mathcal{S}$. We define

$$
(x, \widehat{k}, z) \leq\left(x^{\prime}, \widehat{g}, y^{\prime}\right) ;\left(y^{\prime \prime}, \widehat{h}, z^{\prime \prime}\right)
$$

if the following conditions hold:

- $x=x^{\prime}$,

- $y^{\prime}=y^{\prime \prime}=y$, say,

- $z=z^{\prime \prime}$,

- $g+h+k+c_{x y z} \in L_{x y z \Delta}$.

This is well defined - independent of the choice of representatives $g, h, k$ of the relevant cosets. For example, if $(x, \widehat{k}, z)=\left(x, \widehat{k^{\prime}}, z\right)$, then $L_{x z \Delta}+k=L_{x z \Delta}+k^{\prime}$, so $k+k^{\prime} \in L_{x z \Delta} \subseteq L_{x y z \Delta}$. Hence, $g+h+k+c_{x y z} \in L_{x y z \Delta}$ iff $g+h+k^{\prime}+c_{x y z} \in L_{x y z \Delta}$.

Much of this definition is motivated by [3], including the crucial idea that $c_{p q r} \notin L_{p q r \Delta}$. This gives a 'twist' to composition. Like a wrinkle that stops a carpet being laid flat, it stops $\mathcal{A}^{(d)}$ being representable, but it becomes visible only on fairly large scales, as propositions 3.13 and 3.15 will show.

\section{REMARK 3.3 (on composition)}

1. For $s, t, u \in \mathcal{S}$, we have $s \leq t ; u$ as defined in part 8 above iff in $\mathcal{A}^{(d)}$ we have $\{s\} \leq\{t\} ;\{u\}$, so our use of the symbols $\leq$ and ; in the ternary relation on $\mathcal{S}$ in part 8 is not misleading.

2. If $s, t, u \in \mathcal{S}$ and $s \leq t ; u$, then $\operatorname{start}(s)=\operatorname{start}(t)$, end $(s)=\operatorname{end}(u)$, and end $(t)=$ $\operatorname{start}(u)$. 
3. Composition is additive: $a ;(b+c)=a ; b+a ; c$ and $(a+b) ; c=a ; c+b ; c$ for all $a, b, c$ in $\mathcal{A}^{(d)}$. Hence, it is monotonic: if $a \leq a^{\prime}$ and $b \leq b^{\prime}$ in $\mathcal{A}^{(d)}$ then $a ; b \leq a^{\prime} ; b^{\prime}$.

It is easy to see that $\mathcal{A}^{(d)}$ is a finite, hence complete, non-associative algebra. It is in fact a coset relation algebra, as per [3], but we will not need to prove that here. We will show in corollary 3.14 that it is a relation algebra. The proof of that corollary requires $\mathcal{A}^{(d)}$ to be a weakly associative algebra, so we prove this directly as our next step.

\section{3 $\mathcal{A}^{(d)}$ is a weakly associative algebra}

To clarify the proof of the headline here, we make a quick definition:

DEFINITION 3.4 For $x \in K$ we define the following elements of $\mathcal{A}^{(d)}$ :

- ${ }_{x} \mathcal{S}=\{s \in \mathcal{S}: \operatorname{start}(s)=x\}$,

- $\mathcal{S}_{x}=\{s \in \mathcal{S}: \operatorname{end}(s)=x\}$.

LEMMA 3.5 Let $x \in K$ be arbitrary. Then in $\mathcal{A}^{(d)}$ we have

- $s ; 1={ }_{x} \mathcal{S}$ for each $s \in{ }_{x} \mathcal{S}$,

- $1 ; s=\mathcal{S}_{x}$ for each $s \in \mathcal{S}_{x}$.

Proof. For the first part, let $s=(x, \widehat{g}, y) \in{ }_{x} \mathcal{S}$. We show that $s ; 1={ }_{x} \mathcal{S}$.

So let $(z, \widehat{k}, t) \in \mathcal{S}$ be arbitrary. By definition of composition, $(z, \widehat{k}, t) \in(x, \widehat{g}, y) ; 1$ iff there is $(y, \widehat{h}, t) \in 1=\mathcal{S}$ with $(z, \widehat{k}, t) \leq(x, \widehat{g}, y) ;(y, \widehat{h}, t)$. This holds iff $z=x$ and there is $h \in V$ with $g+h+k+c_{x y t} \in L_{x y t \Delta}$. But there always is such an $h$ - for example, $h=g+k+c_{x y t}$. So $(z, \widehat{k}, t) \leq(x, \widehat{g}, y) ; 1$ iff $z=x$, iff $(z, \widehat{k}, t) \in{ }_{x} \mathcal{S}$.

It follows that $s ; 1={ }_{x} \mathcal{S}$, proving the first part. The second part is similar.

LEMMA 3.6 $\mathcal{A}^{(d)}$ is a weakly associative algebra.

Proof. In any non-associative algebra, we have $1 ; 1=1[19$, theorem $1.13(5)]$. So by additivity of composition, it suffices to show that $(a ; 1) ; 1=a ; 1$ for every atom $a$ of $\mathcal{A}^{(d)}$ with $a \leq 1$ '. Take such an atom, say $a=(x, \widehat{0}, x)$. By lemma $3.5, a ; 1={ }_{x} \mathcal{S}$. By definition of composition and lemma 3.5 again, $(a ; 1) ; 1={ }_{x} \mathcal{S} ; 1=\{s \in \mathcal{S}: s \leq t ; 1$ for some $t \in$ $\left.{ }_{x} \mathcal{S}\right\}=\left\{s \in \mathcal{S}: s \leq{ }_{x} \mathcal{S}\right\}={ }_{x} \mathcal{S}=a ; 1$. 


\subsection{Networks}

In the next three subsections, we mostly recall known material due to Maddux. For the first two of them, let $3 \leq m<\omega$. We take $m$ to be the ordinal $\{0,1, \ldots, m-1\}$ of course. Let $\mathcal{B}$ be an atomic non-associative algebra. We write At $\mathcal{B}$ for the set of atoms of $\mathcal{B}$.

DEFINITION 3.7 An $m$-dimensional (atomic) network over $\mathcal{B}$ is a map $N: m \times m \rightarrow$ At $\mathcal{B}$ satisfying, for every $x, y, z<m$,

1. $N(x, x) \leq 1^{\prime}$,

2. $N(x, y)=N(y, x)^{\cup}$,

3. $N(x, z) \leq N(x, y) ; N(y, z)$.

For $m$-dimensional atomic networks $M, N$ over $\mathcal{B}$, and $x, y<m$, we write $M \equiv_{x} N$ (respectively, $\left.M \equiv_{x y} N\right)$ if $M(z, t)=N(z, t)$ for every $z, t \in m \backslash\{x\}$ (respectively, every $z, t \in m \backslash\{x, y\})$. In Maddux's language, $M$ and $N$ agree off of $x$ (or $x, y$ ).

Our algebra $\mathcal{A}^{(d)}$ is an atomic non-associative algebra, so the notion of an $m$-dimensional atomic network over $\mathcal{A}^{(d)}$ is defined. As we now see, each 'node' in such a network has a 'location' in $K$ which is the start or end of all atoms associated with it.

DEFINITION 3.8 Let $N$ be an $m$-dimensional atomic network over $\mathcal{A}^{(d)}$. We define a $\operatorname{map} \nu_{N}: m \rightarrow K$ by

$$
\nu_{N}(x)=\operatorname{start}(N(x, x)), \quad \text { for each } x<m .
$$

Since $N(x, x) \leq 1$, we also have $\nu_{N}(x)=\operatorname{end}(N(x, x))$ and $N(x, x)=\left(\nu_{N}(x), \widehat{0}, \nu_{N}(x)\right)$. The element $\nu_{N}(x)$ is in some sense the location in $K$ of the node $x$.

LEMMA 3.9 Let $N$ be an m-dimensional atomic network over $\mathcal{A}^{(d)}$. Then for every $x, y<m$, we have $N(x, y)=\left(\nu_{N}(x), \widehat{v}, \nu_{N}(y)\right)$ for some $v \in V$.

Proof. As $N$ is a network, $N(x, x) \leq N(x, y) ; N(y, x)$. Remark 3.3 now yields that $\operatorname{start}(N(x, y))=\operatorname{start}(N(x, x))=\nu_{N}(x)$. So $\operatorname{end}(N(x, y))=\operatorname{start}(N(y, x))=\nu_{N}(y)$ by this and the remark.

\subsection{Bases}

We now define two kinds of 'basis' of $\mathcal{B}$. We give references to papers, but the definitions can also be found in $[23, \S 21]$ and $[13$, chapter 12$]$. Let $\mathcal{M}$ be a set of $m$-dimensional atomic networks over $\mathcal{B}$.

DEFINITION 3.10 (Maddux, [20, p.78], [21, definition 3]) We say that $\mathcal{M}$ is an $m$-dimensional relational basis of $\mathcal{B}$ if:

Australasian Journal of Logic (17:2) 2020, Article no. 1 
1. for every $a \in$ At $\mathcal{B}$, there is $N \in \mathcal{M}$ with $N(0,1)=a$,

2. for every $x, y, z<m$ with $z \neq x, y$, every $N \in \mathcal{M}$, and every $a, b \in$ At $\mathcal{B}$ with $N(x, y) \leq a ; b$, there is $M \in \mathcal{M}$ with $M \equiv_{z} N, M(x, z)=a$, and $M(z, y)=b$. This is called the triangle addition property.

DEFINITION 3.11 (Maddux, [21, definition 4]) We say that $\mathcal{M}$ is an m-dimensional cylindric basis of $\mathcal{B}$ if:

1. For every $a, b, c \in$ At $\mathcal{B}$ with $a \leq b ; c$, there is $N \in \mathcal{M}$ with $N(0,1)=a, N(0,2)=b$, and $N(2,1)=c$.

2. Let $x, y<m$. For each $z<m$, define

$$
z^{\prime}= \begin{cases}y, & \text { if } z=x, \\ z, & \text { otherwise }\end{cases}
$$

Let $N \in \mathcal{M}$ be arbitrary, and let $N^{\prime}: m^{2} \rightarrow$ At $\mathcal{B}$ be given by $N^{\prime}(z, t)=N\left(z^{\prime}, t^{\prime}\right)$. Then $N^{\prime} \in \mathcal{M}$. $\left(N^{\prime}\right.$ is often written as $N[x / y]$.)

3. If $x, y<m$ are distinct and $N, M \in \mathcal{M}$ satisfy $N \equiv_{x y} M$, then there is some $L \in \mathcal{M}$ with $N \equiv_{x} L \equiv_{y} M$. This is called the amalgamation property.

Any $m$-dimensional cylindric basis of an atomic weakly associative algebra is also an $m$ dimensional relational basis [23, theorem 334(ii)]. Weak associativity is essential here [13, exercise $12.6(5)$ ], which is why we proved that $\mathcal{A}^{(d)}$ is weakly associative, in lemma 3.6.

\subsection{Some varieties of relation algebras}

We will be using the classes $\mathrm{RA}_{m}$ of relation algebras, for finite $m \geq 3$. $\mathrm{RA}_{m}$ is the closure under subalgebras of the class of complete atomic non-associative algebras that have an $m$-dimensional relational basis ([21, definition 3(ii-iii)] or $[23, \S 24])$. We will need only the following facts.

FACT 3.12 Suppose that $3 \leq m<\omega$.

1. $\mathrm{RA}_{m+1} \subseteq \mathrm{RA}_{m}[20$, theorem 3].

2. $\mathrm{RA}_{4}=\mathrm{RA}[20$, theorem $6(2)]$.

3. $\mathrm{RRA}=\bigcap_{3 \leq m<\omega} \mathrm{RA}_{m}[20$, theorems 6(3) and 10].

4. $\mathrm{RA}_{m}$ is a variety $[20$, theorem 9$]$.

5. If $\mathcal{B}$ is a finite non-associative algebra, then $\mathcal{B} \in \mathrm{RA}_{m}$ iff $\mathcal{B}$ has an $m$-dimensional relational basis $\left(\Leftarrow\right.$ is by definition of $\mathrm{RA}_{m}$, and $\Rightarrow$ follows from [20, theorem 8$]$ ).

The two main results of this section will show that $\mathcal{A}^{(d)}$ has a $(d+3)$-dimensional cylindric basis but no $(d+4)$-dimensional relational basis. It will follow that $\mathcal{A}^{(d)} \in \mathrm{RA}_{d+3} \backslash \mathrm{RA}_{d+4}$.

Australasian Journal of Logic (17:2) 2020, Article no. 1 


\section{7 $\mathcal{A}^{(d)}$ has a $(d+3)$-dimensional cylindric basis}

PROPOSITION 3.13 The set $\mathcal{M}$ of all $(d+3)$-dimensional atomic networks over $\mathcal{A}^{(d)}$ is a $(d+3)$-dimensional cylindric basis of $\mathcal{A}^{(d)}$.

Proof. The first two properties defining a cylindric basis actually hold for every atomic weakly associative algebra when $\mathcal{M}$ is taken to be the set of all atomic networks of appropriate dimension, as it is here. But we will check them anyway.

First let $s, t, u \in \mathcal{S}$ with $s \leq t ; u$. We will define $N \in \mathcal{M}$ with $N(0,1)=s, N(0,2)=t$, and $N(2,1)=u$.

We start out by defining the part of $N$ on the indices $0,1,2$. Of course we define $N(0,1)=s, N(0,2)=t$, and $N(2,1)=u$. We put $N(1,0)=\breve{s}, N(2,0)=\breve{t}$, and $N(1,2)=\breve{u}$. We also put $N(0,0)=(\operatorname{start}(s), \widehat{0}, \operatorname{start}(s)), N(1,1)=(\operatorname{end}(s), \widehat{0}, \operatorname{end}(s))$, and $N(2,2)=(\operatorname{end}(t), \widehat{0}$, end $(t))$ - note that end $(t)=\operatorname{start}(u)$, by remark 3.3. Extend the definition of $N$ to all indices $x, y<d+3$ by putting $N(x, y)=N(\min (x, 2), \min (y, 2))$, which we have already defined. It is easily checked that $N \in \mathcal{M}$ and is as required.

It is extremely easy to check that if $N \in \mathcal{M}$ then the map $N^{\prime}$ in definition 3.11 is an atomic network, so is in $\mathcal{M}$ as well.

We come to the amalgamation property now. So let $x, y<d+3$ be distinct and let $N, M \in \mathcal{M}$ satisfy $N \equiv_{x y} M$. We will define $L \in \mathcal{M}$ with $N \equiv_{x} L \equiv_{y} M$. See figure 1 .

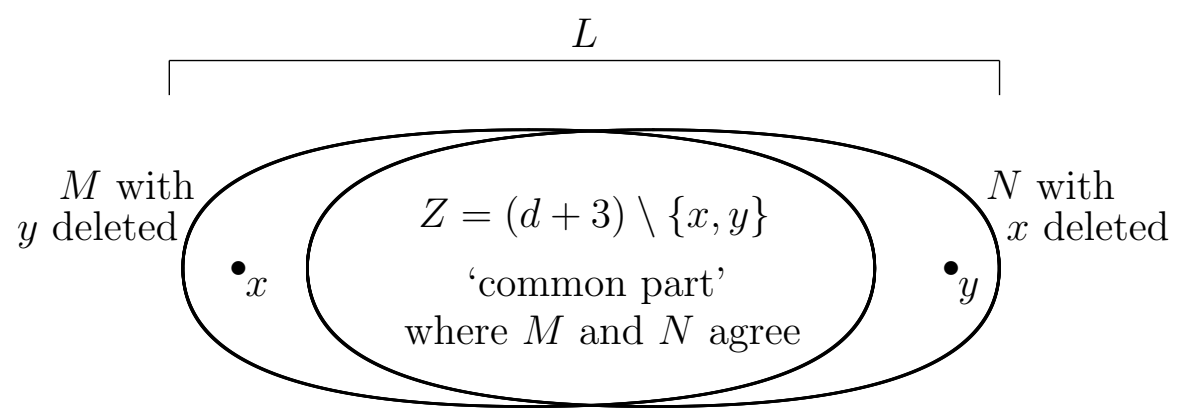

Figure 1: Amalgamation property

We need to define the atom $L(z, t) \in \mathcal{S}$ for every $z, t<d+3$. Now $L(z, t)$ is already determined whenever $\{z, t\} \subseteq(d+3) \backslash\{x\}$, because we need $L \equiv_{x} N$, so we have to put $L(z, t)=N(z, t)$ in this case. Similarly, $L(z, t)$ is determined whenever $\{z, t\} \subseteq(d+3) \backslash\{y\}$ : we need $L \equiv_{y} M$, so we have to put $L(z, t)=M(z, t)$. This is well defined when $\{z, t\} \subseteq$ $(d+3) \backslash\{x, y\}$, since $M \equiv_{x y} N$.

The remaining case is when $x, y \in\{z, t\}$, so since $x \neq y$, we have $\{z, t\}=\{x, y\}$. We will define $L(x, y)$, and then let $L(y, x)$ be its converse.

Recall from definition 3.8 that $\nu_{N}(t)=\operatorname{start}(N(t, t)) \in K$, and similarly for $M$. For each $t<d+3$, write

$$
\check{t}= \begin{cases}\nu_{N}(t), & \text { if } t \neq x, \\ \nu_{M}(t), & \text { if } t \neq y .\end{cases}
$$

Australasian Journal of Logic (17:2) 2020, Article no. 1 
Since $N \equiv_{x y} M$, if $t \neq x, y$ then $\nu_{M}(t)=\nu_{N}(t)$, so this is well defined (we will have $\left.\check{t}=\nu_{L}(t)\right)$.

Let $Z=(d+3) \backslash\{x, y\}$, the 'common part' in figure 1. Since $M$ and $N$ are networks, it follows from lemma 3.9 that for each $z \in Z$ we can choose $g_{z}, h_{z} \in V$ such that

$$
\begin{aligned}
& L(x, z)=M(x, z)=\left(\check{x}, \widehat{g_{z}}, \check{z}\right) \\
& L(z, y)=N(z, y)=\left(\check{z}, \widehat{h_{z}}, \check{y}\right) .
\end{aligned}
$$

We need to define $L(x, y)=(\check{x}, \widehat{k}, \check{y})$ for some $k \in V$ with $(\check{x}, \widehat{k}, \check{y}) \leq\left(\check{x}, \widehat{g_{z}}, \check{z}\right) ;\left(\check{z}, \widehat{h_{z}}, \check{y}\right)$ for each $z \in Z$, so that $L(x, y) \leq L(x, z) ; L(z, y)$. So by definition of composition in $\mathcal{A}^{(d)}$, we need to find $k \in V$ such that

$$
k+g_{z}+h_{z}+c_{\check{x} \check{y} \check{z}} \in L_{\check{x} \check{y} \check{z} \Delta} \quad \text { for each } z \in Z \text {. }
$$

The way we do it is one of the two critical points of the paper.

First, we define a field element $\lambda_{Y} \in \mathcal{F}$ for each $Y \in K^{[d+1]}$ with $\check{x}, \check{y} \notin Y$, as follows.

1. If there is $z \in Z$ with $\check{z} \notin Y$, then we put $\lambda_{Y}=\left(g_{z}+h_{z}+c_{\check{x} \check{y} \check{z}}\right) \cdot \chi_{Y \downarrow}$.

2. If there is no such $z$, we put $\lambda_{Y}=0$ (actually, any value will do).

Claim. $\lambda_{Y}$ is well defined.

Proof of claim. Take $Y \in K^{[d+1]}$ with $\check{x}, \check{y} \notin Y$. Suppose that $z, z^{\prime} \in Z, z \neq z^{\prime}$, and $\check{z}, \check{z} \prime \notin Y$. We need to show that

$$
\left(g_{z}+h_{z}+c_{\check{x} \check{y} \check{z}}\right) \cdot \chi_{Y \downarrow}=\left(g_{z^{\prime}}+h_{z^{\prime}}+c_{\check{x} \check{y} \check{z}^{\prime}}\right) \cdot \chi_{Y \downarrow} .
$$

It will be useful to define

$$
S=\{\check{x}, \check{y}, \check{z}, \check{z} \prime\} \subseteq K \backslash Y .
$$

Let $M\left(z, z^{\prime}\right)=N\left(z, z^{\prime}\right)=\left(\check{z}, \widehat{w}, \check{z}^{\prime}\right)$, say, where $w \in V$. The value is the same in $M$ and $N$ because $M \equiv_{x y} N$ and $z, z^{\prime} \notin\{x, y\}$. Since $M$ is a network, we have $M\left(z, z^{\prime}\right) \leq$ $M(z, x) ; M\left(x, z^{\prime}\right)$, and from this and (3) we obtain $g_{z}+g_{z^{\prime}}+w+c_{\check{x} \check{z} z^{\prime}} \in L_{\check{x} \check{z} \check{z}^{\prime} \Delta} \subseteq L_{S \cup\{\Delta\}}$. On the other side, since $N$ is a network, $N\left(z, z^{\prime}\right) \leq N(z, y) ; N\left(y, z^{\prime}\right)$, which with (3) gives $h_{z}+h_{z^{\prime}}+w+c_{\check{y} \check{z} z^{\prime}} \in L_{\check{y} \check{z} \check{z}^{\prime} \Delta} \subseteq L_{S \cup\{\Delta\}}$. Adding these yields

$$
g_{z}+g_{z^{\prime}}+w+c_{\check{x} \check{z} \check{z}^{\prime}}+h_{z}+h_{z^{\prime}}+w+c_{\check{y} \check{z} \check{z}^{\prime}} \in L_{S \cup\{\Delta\}} .
$$

Bear in mind that $\mathcal{F}$ has characteristic 2. So the two $w$ here cancel, and by adding $c_{\breve{x} \check{y} \check{z}}+c_{\check{x} \check{y} z^{\prime}}+c_{\check{x} \check{y} \check{z}}+c_{\check{x} \check{y} \check{z}^{\prime}}=0$ and rearranging, we get

$$
\left(g_{z}+h_{z}+c_{\check{x} \check{y} \check{z}}\right)+\left(g_{z^{\prime}}+h_{z^{\prime}}+c_{\check{x} \check{y} \check{z}^{\prime}}\right)+\underbrace{\left(c_{\check{x} \check{z} \check{z^{\prime}}}+c_{\check{y} \check{z} \check{z^{\prime}}}+c_{\check{x} \check{y} \check{z}}+c_{\check{x} \check{y} \check{z}^{\prime}}\right)}_{v, \text { say }} \in L_{S \cup\{\Delta\}} .
$$

We will show that the vector $v$ indicated in (6) is zero. Put $I=\left\{x, y, z, z^{\prime}\right\}$, so that $S=\{\check{i}: i \in I\}$. Define a map $f: I \rightarrow \wp(S)$ by $f(i)=\{\check{j}: j \in I \backslash\{i\}\}$. So

$$
v \stackrel{\text { def }}{=} c_{\check{x} \check{z} \check{z^{\prime}}}+c_{\check{y} \check{z} \check{z^{\prime}}}+c_{\check{x} \check{y} \check{z}}+c_{\check{x} \check{y} \bar{z}^{\prime}}=c_{f(y)}+c_{f(x)}+c_{f\left(z^{\prime}\right)}+c_{f(z)}=\sum_{i \in I} c_{f(i)} .
$$

Australasian Journal of Logic (17:2) 2020, Article no. 1 
Now observe that $S \subseteq K \backslash Y$ and $|K \backslash Y|=|K|-|Y|=(d+4)-(d+1)=3$. So, crucially, $|S| \leq 3$, while $|I|=4$ since we assumed $z \neq z^{\prime}$. So by the pigeonhole principle, there are distinct $i, j \in I$ with $\breve{i}=\breve{j}$. It follows that $f(i)=f(j)=S$, and that $|f(k)| \leq 2$ for each $k \in I \backslash\{i, j\}$, so $c_{f(k)}=0$ by definition $3.2(2)$. So indeed,

$$
v=\sum_{i^{\prime} \in I} c_{f\left(i^{\prime}\right)}=c_{S}+c_{S}+0+0=0
$$

As a result, (6) reduces to

$$
\left(g_{z}+h_{z}+c_{\check{x} \check{y} \check{z}}\right)+\left(g_{z^{\prime}}+h_{z^{\prime}}+c_{\check{x} \check{y} \check{z}^{\prime}}\right) \in L_{S \cup\{\Delta\}} .
$$

As $Y$ is disjoint from $S$, by proposition $2.12(\mathrm{~L} 1 \Rightarrow \mathrm{L} 2)$ we obtain

$$
\left(\left(g_{z}+h_{z}+c_{\check{x} \check{y} \check{z}}\right)+\left(g_{z^{\prime}}+h_{z^{\prime}}+c_{\check{x} \check{y} \check{z}^{\prime}}\right)\right) \cdot \chi_{Y \downarrow}=0 .
$$

By linearity of scalar product, this yields

$$
\left(g_{z}+h_{z}+c_{\check{x} \check{y} \check{z}}\right) \cdot \chi_{Y \downarrow}=\left(g_{z^{\prime}}+h_{z^{\prime}}+c_{\check{x} \check{y} \check{z}^{\prime}}\right) \cdot \chi_{Y \downarrow},
$$

proving (5), and with it, the claim.

We now have a clear run to the finish. Since $|\{\check{t}: t<d+3\}|<d+4=|K|$, we can select some $a \in K \backslash\{\check{t}: t<d+3\}$. We now define our $k \in V$ by

$$
k(e)= \begin{cases}\lambda_{e \cup\{a\}}, & \text { if } \check{x}, \check{y}, a \notin e, \\ 0, & \text { otherwise }\end{cases}
$$

for $e \in E$. We check that (4) holds. So fix arbitrary $z \in Z$; we use proposition 2.12 to

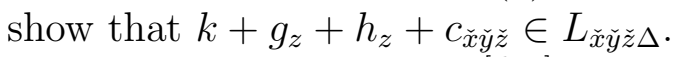

To this end, let $Y \in K^{[d+1]}$ be disjoint from $\{\check{x}, \check{y}, \check{z}\}$ and contain $a$. The edges in $Y \downarrow$ are $Y \backslash\{a\}$, on which $k$ takes the value $\lambda_{Y}$, and a bunch of edges containing $a$, on which $k$ takes the value 0 . The sum of these values is $\lambda_{Y}$. So by definition of scalar product and of $\lambda_{Y}$,

$$
k \cdot \chi_{Y \downarrow}=\sum_{e \in Y \downarrow} k(e)=\lambda_{Y}=\left(g_{z}+h_{z}+c_{\check{x} \check{y} \check{z}}\right) \cdot \chi_{Y \downarrow} .
$$

So by linearity of scalar product and the characteristic of $\mathcal{F}$ as usual,

$$
\left(k+g_{z}+h_{z}+c_{\check{x} \check{y} \check{z}}\right) \cdot \chi_{Y \downarrow}=k \cdot \chi_{Y \downarrow}+\left(g_{z}+h_{z}+c_{\check{x} \check{y} \check{z}}\right) \cdot \chi_{Y \downarrow}=k \cdot \chi_{Y \downarrow}+k \cdot \chi_{Y \downarrow}=0 .
$$

This holds for every such $Y$, so since $a \notin\{\check{x}, \check{y}, \check{z}\}$, by proposition 2.12 (L3 $\Rightarrow$ L1) we obtain

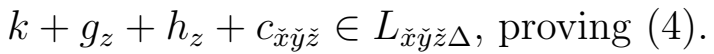

Now, defining $L(x, y)=(\check{x}, \widehat{k}, \check{y})$ and $L(y, x)=(\check{y}, \widehat{k}, \check{x})$ as intimated earlier, it is straightforward to check that $L \in \mathcal{M}$, and plainly $N \equiv_{x} L \equiv_{y} M$. 
COROLLARY $3.14 \mathcal{A}^{(d)} \in \mathrm{RA}_{d+3}$. Hence, $\mathcal{A}^{(d)}$ is a relation algebra.

Proof. By proposition 3.13, $\mathcal{A}^{(d)}$ has a $(d+3)$-dimensional cylindric basis, $\mathcal{M}$. By lemma $3.6, \mathcal{A}^{(d)}$ is a weakly associative algebra, so by [23, theorem 334(ii)], already mentioned, $\mathcal{M}$ is also a $(d+3)$-dimensional relational basis of $\mathcal{A}^{(d)}$. By fact 3.12 and since $d+3 \geq 4$, we obtain $\mathcal{A}^{(d)} \in \mathrm{RA}_{d+3} \subseteq \mathrm{RA}_{4}=\mathrm{RA}$.

We can show a little more for those interested. Since $\mathcal{A}^{(d)}$ is finite, it is complete. So $[21$, theorem $10(\mathrm{i}, \mathrm{iii})]$ applies, showing that there is an atomic $(d+3)$-dimensional cylindric algebra $\mathcal{C}$ such that $\mathcal{A}^{(d)}$ is isomorphic to the relation algebra reduct of $\mathcal{C}$. (Actually, $\mathcal{C}$ is the standard complex algebra over the cylindric basis $\mathcal{M}$, and is therefore finite.) So in fact, $\mathcal{A}^{(d)} \in \mathfrak{R a C A}{ }_{d+3}$, a proper subclass of $\mathrm{RA}_{d+3}[12]$.

\section{8 $\mathcal{A}^{(d)}$ has no $(d+4)$-dimensional relational basis}

We now come to the second critical point of the paper. It is where the wrinkle mentioned before shows up.

PROPOSITION 3.15 $\mathcal{A}^{(d)}$ has no $(d+4)$-dimensional relational basis.

Proof. Suppose for contradiction that $\mathcal{M}$ is a $(d+4)$-dimensional relational basis for $\mathcal{A}^{(d)}$. We will find a network $M \in \mathcal{M}$ that is 'full' in the sense that $\nu_{M}:(d+4) \rightarrow K$ is surjective - every possible 'location' appears in $M$. We will then obtain our contradiction by showing that no such 'full' network can exist.

Enumerate $K$ as $\left\{x_{i}: i<d+4\right\}$. We will define networks $M_{j} \in \mathcal{M}$ for $j=1, \ldots, d+3$ by induction, such that

$$
\nu_{M_{j}}(i)=x_{i} \quad \text { for each } i=0, \ldots, j .
$$

As $\mathcal{M}$ is a relational basis, there is $M_{1} \in \mathcal{M}$ with $M_{1}(0,1)=\left(x_{0}, \widehat{0}, x_{1}\right)$. By lemma 3.9, $\nu_{M_{1}}(0)=x_{0}$ and $\nu_{M_{1}}(1)=x_{1}$, so $M_{1}$ satisfies $(7)$.

Let $1 \leq j<d+3$ and assume inductively that $M_{j}$ has been defined. So from (7) and because $M_{j}(0,0) \leq 1$, we have $M_{j}(0,0)=\left(\nu_{M_{j}}(0), \widehat{0}, \nu_{M_{j}}(0)\right)=\left(x_{0}, \widehat{0}, x_{0}\right)$. It follows by definition of composition in $\mathcal{A}^{(d)}$ that

$$
M_{j}(0,0)=\left(x_{0}, \widehat{0}, x_{0}\right) \leq\left(x_{0}, \widehat{0}, x_{j+1}\right) ;\left(x_{j+1}, \widehat{0}, x_{0}\right),
$$

since $c_{x_{0} x_{j+1} x_{0}}=0$ by definition $3.2(2)$, so $0+0+0+c_{x_{0} x_{j+1} x_{0}}=0 \in L_{x_{0} x_{j+1} \Delta}$. So by the triangle addition property of $\mathcal{M}$, there is $M_{j+1} \in \mathcal{M}$ with $M_{j+1} \equiv_{j+1} M_{j}$ and $M_{j+1}(0, j+1)=\left(x_{0}, \widehat{0}, x_{j+1}\right)$. So $\nu_{M_{j+1}}(j+1)=x_{j+1}$; and since $M_{j+1} \equiv_{j+1} M_{j}$, which inductively satisfies (7), for each $i \leq j$ we have $\nu_{M_{j+1}}(i)=\nu_{M_{j}}(i)=x_{i}$. It follows that $M_{j+1}$ satisfies (7) as well. This completes the induction.

From now on, we write $M$ for $M_{d+3} \in \mathcal{M}$. By $(7), \nu_{M}(i)=x_{i}$ for each $i<d+4$. So $M$ is 'full' in the sense described above, and indeed, $\nu_{M}:(d+4) \rightarrow K$ is a bijection. 
Using that $\nu_{M}$ is bijective, we can define a map $N: K^{[1]} \cup K^{[2]} \rightarrow V$ such that

$$
\left.M\left(\nu_{M}^{-1}(x), \nu_{M}^{-1}(y)\right)=(x, N \widehat{(\{x, y\}}), y\right) \quad \text { for every } x, y \in K .
$$

It can be done as follows. For each $i \leq j<d+4$, we have $M(i, j)=\left(\nu_{M}(i), \widehat{v}, \nu_{M}(j)\right)$ for some (not unique) $v \in V$. Choose such a $v$, and define $N\left(\left\{\nu_{M}(i), \nu_{M}(j)\right\}\right)=v$. Since $M(j, i)=M(i, j)^{\cup}=\left(\nu_{M}(j), \widehat{v}, \nu_{M}(i)\right)$ for the same $v$, we can see that (8) holds. For brevity, for $x, y \in K$ we will often write $N(\{x, y\})$ simply as $N(x, y)$. Obviously, $N(x, y)=N(y, x)$.

Since $M$ is a network, it follows from definition 3.2(8) that

$$
N(x, y)+N(y, z)+N(z, x)+c_{x y z} \in L_{x y z \Delta} \quad \text { for every } x, y, z \in K .
$$

Take any $S=\{x, y, z\} \in K^{[3]}$. Define

$$
T_{S} \stackrel{\text { def }}{=} \sum\{N(x, y)(e)+N(y, z)(e)+N(z, x)(e): e \in E, x, y, z \notin e\} \in \mathcal{F} .
$$

This is independent of the choice of enumeration of $S$ as $\{x, y, z\}$, so is well defined. Since $|K|=d+4$, the complement $K \backslash S$ of $S$ has size $d+1$, and hence $K \backslash S$ is the unique $Y \in K^{[d+1]}$ disjoint from $S$. So for each $v \in V$ we have

$$
\begin{aligned}
v \in L_{x y z \Delta} & \Longleftrightarrow v \cdot \chi_{(K \backslash S) \downarrow}=0 & & \text { by proposition 2.12(L1 } \Leftrightarrow \mathrm{L} 2) \\
& \Longleftrightarrow \sum\{v(e): e \in E, x, y, z \notin e\}=0 & & \text { by evaluating. }
\end{aligned}
$$

Applying this with $v=N(x, y)+N(y, z)+N(z, x)$ gives

$$
N(x, y)+N(y, z)+N(z, x) \in L_{x y z \Delta} \Longleftrightarrow T_{S}=0 .
$$

Put $S_{0}=\{p, q, r\}$ (see definition 3.2(1) for $\left.p, q, r\right)$. Now if $S \neq S_{0}$ then $c_{S}=0$ by definition $3.2(2)$, so by (9), the left-hand side of (10) holds. We deduce from (10) that

$$
T_{S}=0 \text { for every } S \in K^{[3]} \backslash\left\{S_{0}\right\} \text {. }
$$

Hence,

$$
\mathcal{T} \stackrel{\text { def }}{=} \sum_{S \in K^{[3]}} T_{S}=T_{S_{0}}
$$

But let us inspect the sum $\mathcal{T}$ of all $T_{S}$ in its own right. Consider a term $N(\{x, y\})(e)$ in this sum. It comes from one or more of the $T_{S}$ whose sum is $\mathcal{T}$. In fact, a little thought shows that this term occurs once in each $T_{S}$ with $S \in K^{[3]}$ disjoint from $e$ and containing $x, y$, and not at all in any other $T_{S}$. Now $|K|=d+4$ and $|e|=d$. So $|K \backslash e|=4$, and hence there are distinct $t, u \in K$ such that $K=e \cup\{x, y, t, u\}$. Then $\{x, y, t\}$ and $\{x, y, u\}$ are the only sets in $K^{[3]}$ containing $x$ and $y$ and disjoint from $e$. So $N(\{x, y\})(e)$ occurs precisely twice in $\mathcal{T}$ : once in $T_{\{x, y, t\}}$ and once in $T_{\{x, y, u\}}$. Since $\mathcal{F}$ has characteristic 2 , the sum of these two occurrences is zero. This holds for all terms in $\mathcal{T}$ - they all occur in pairs and cancel. So we obtain $\mathcal{T}=0$.

We conclude from this and (11) that $T_{S_{0}}=0$. So by (10) again, $N(p, q)+N(q, r)+$ $N(r, p) \in L_{p q r \Delta}$. Now (9) yields that $c_{p q r} \in L_{p q r \Delta}$ as well, contradicting the original (wrinkly) choice of $c_{p q r} \notin L_{p q r \Delta}$ in definition 3.2(2).

Australasian Journal of Logic (17:2) 2020, Article no. 1 
COROLLARY 3.16 $\mathcal{A}^{(d)} \notin \mathrm{RA}_{d+4}$. Hence, $\mathcal{A}^{(d)}$ is not representable.

Proof. By proposition $3.15, \mathcal{A}^{(d)}$ has no $(d+4)$-dimensional relational basis. We saw in fact 3.12(5) that every finite algebra in $\mathrm{RA}_{d+4}$ actually has a $(d+4)$-dimensional relational basis of its own. Since $\mathcal{A}^{(d)}$ is finite, it follows that $\mathcal{A}^{(d)} \notin \mathrm{RA}_{d+4}$. Since RRA $\subseteq \mathrm{RA}_{d+4}$, we obtain $\mathcal{A}^{(d)} \notin \mathrm{RRA}$, so $\mathcal{A}^{(d)}$ is not representable.

\section{$3.9 \mathcal{A}^{(d)}$ is simple, measurable, and persistently finite}

Now that we know (from corollary 3.14) that $\mathcal{A}^{(d)}$ is a relation algebra, we can establish three more of its basic properties.

As is standard in algebra, a relation algebra $\mathcal{A}$ is said to be simple if it is nontrivial and, up to isomorphism, its only homomorphic images are itself and the trivial one-element algebra. A nontrivial relation algebra $\mathcal{A}$ is simple iff $1 ; a ; 1=1$ for every non-zero element $a$ of $\mathcal{A}$ [23, theorem 379]. So RA is a discriminator variety: it has the unary discriminator term $1 ; x ; 1$. For relation algebras, simplicity is defined by the universal first-order sentence $0 \neq 1 \wedge \forall x(x \neq 0 \rightarrow 1 ; x ; 1=1)$, and it follows that every subalgebra of a simple relation algebra is also simple.

LEMMA $3.17 \mathcal{A}^{(d)}$ is simple.

Proof. By the above, it is enough to show that $1 ; a ; 1=1$ for each nonzero element $a$ of $\mathcal{A}^{(d)}$, and by monotonicity of composition, we can take $a$ to be an atom. By lemma 3.5, $1 ; a=\mathcal{S}_{\operatorname{end}(a)}$, and so $1 ; a ; 1=\mathcal{S}_{\operatorname{end}(a)} ; 1=\bigcup_{b \in \mathcal{S}_{\text {end }(a)}}(b ; 1)=\bigcup_{\operatorname{end}(b)=\operatorname{end}(a) \operatorname{start}(b)} \mathcal{S}=$ $\bigcup_{z \in K} \mathcal{S}=\mathcal{S}=1$.

An element $a$ of a relation algebra $\mathcal{A}$ is said to be functional if $\breve{a} ; a \leq 1$, and an identity atom if it is an atom of $\mathcal{A}$ and $a \leq 1$ '. We say that $\mathcal{A}$ is measurable if 1 ' is a sum of atoms, and for each identity atom $a$, the element $a ; 1 ; a$ is a sum of functional elements.

LEMMA $3.18 \mathcal{A}^{(d)}$ is measurable.

Proof. Certainly, 1 ' is a sum of atoms since $\mathcal{A}^{(d)}$ is atomic. Take an identity atom $a=$ $(x, \widehat{0}, x)$ of $\mathcal{A}^{(d)}$. Plainly,

$$
\begin{aligned}
a ; 1 ; a & =\{(u, \widehat{g}, v):(u, \widehat{g}, v) \leq(x, \widehat{0}, x) ;(y, \widehat{h}, z) ;(x, \widehat{0}, x) \text { for some }(y, \widehat{h}, z) \in \mathcal{S}\} \\
& =\{(x, \widehat{h}, x): h \in V\} .
\end{aligned}
$$

This is a sum of atoms of the form $(x, \widehat{h}, x)$. Each such atom is functional, because it is self-converse, and for any $(y, \widehat{g}, z) \in \mathcal{S}$ we have

$$
\begin{aligned}
(y, \widehat{g}, z) \leq(x, \widehat{h}, x) ;(x, \widehat{h}, x) & \Longleftrightarrow y=z=x \text { and } g+h+h+c_{x x x}=g \in L_{x \Delta} \\
& \Longleftrightarrow(y, \widehat{g}, z)=(x, \widehat{0}, x) .
\end{aligned}
$$

So $(x, \widehat{h}, x)^{\cup} ;(x, \widehat{h}, x)=(x, \widehat{0}, x) \leq 1$.

Australasian Journal of Logic (17:2) 2020, Article no. 1 
A relation algebra $\mathcal{A}$ is said to be persistently finite (in RA) if $\mathcal{A}$ is finite and every simple relation algebra extending $\mathcal{A}$ is finite as well [2, p.3]. Under this definition, every finite non-simple relation algebra $\mathcal{A}$ is vacuously persistently finite, since as we said above, every subalgebra of a simple relation algebra is simple, and so $\mathcal{A}$ has no simple relation algebra extensions at all. So we are mainly interested in simple persistently finite relation algebras. As we said in the introduction, non-representable examples were constructed in [3]. Additional examples are the simple relation algebras with no proper simple extensions. The representable ones are precisely the algebras isomorphic to $\mathfrak{R e}(U)$ for a finite set $U$ - for $\Leftarrow$ see [18, lemma 7.4], and $\Rightarrow$ is an exercise — and non-representable examples were given in [4], as mentioned in the introduction. We remark that a relation algebra is persistently finite iff it is finite and, up to isomorphism, it has only finitely many simple extensions [2, p. 4].

LEMMA 3.19 $\mathcal{A}^{(d)}$ is persistently finite.

Proof. By the non-trivial [2, theorem 3.1], if $\mathcal{A}$ is a finite measurable relation algebra, $\mathcal{B}$ is a simple relation algebra, and $\mathcal{A} \subseteq \mathcal{B}$, then $\mathcal{B}$ is finite (and measurable). So every finite measurable relation algebra is persistently finite. Since $\mathcal{A}^{(d)}$ is finite, and by lemma 3.18, measurable, it is persistently finite.

\section{Main results}

Having defined our relation algebras $\mathcal{A}^{(d)}$ and established some of their properties, we will soon be able to prove our main results. They involve completions, which we now recall.

\subsection{Completions of relation algebras; the variety $\mathcal{C}(\mathrm{RRA})$}

For relation algebras $\mathcal{A}, \mathcal{B}$, we say that $\mathcal{A}$ is a dense subalgebra of $\mathcal{B}$, and that $\mathcal{B}$ is a dense extension of $\mathcal{A}$, if $\mathcal{A}$ is a subalgebra of $\mathcal{B}$ and for every non-zero element $b$ of $\mathcal{B}$, there is a non-zero element $a$ of $\mathcal{A}$ with $a \leq b$.

A completion of a relation algebra $\mathcal{A}$ is a complete relation algebra (see §3.1) that is a dense extension of $\mathcal{A}$. Monk proved in [26] that every relation algebra $\mathcal{A}$ has a completion, which is unique up to isomorphism over $\mathcal{A}$. So we are justified in speaking of 'the' completion of a relation algebra $\mathcal{A}$, and we write it as $\mathcal{A}^{c}$.

For a class $\mathrm{K}$ of relation algebras, we write $\mathrm{K}^{c}=\left\{\mathcal{A}^{c}: \mathcal{A} \in \mathrm{K}\right\}$, and Var $\mathrm{K}$ for the variety generated by (i.e., the smallest variety containing) $\mathrm{K}$.

DEFINITION 4.1 Let $\mathcal{C}($ RRA) be the variety defined by all equations $\varepsilon$ in the signature of relation algebras that are valid in RRA and preserved by completions of relation algebras — that is, if $\mathcal{A}$ is a relation algebra and $\mathcal{A} \models \varepsilon$, then $\mathcal{A}^{c} \models \varepsilon$.

Here are some observations about $\mathcal{C}(\mathrm{RRA})$. They are easy to prove.

1. $R R A \subseteq \mathcal{C}(R R A)$.

Australasian Journal of Logic (17:2) 2020, Article no. 1 
2. Each of the equations defining the class RA of relation algebras is valid in RRA, and obviously preserved by completions of relation algebras since the completion of a relation algebra is a relation algebra. So $\mathcal{C}(R R A) \subseteq R A$.

3. $\mathcal{C}(\mathrm{RRA})$ is closed under completions: $\mathcal{C}(\mathrm{RRA})^{c} \subseteq \mathcal{C}(\mathrm{RRA})$.

Our first main result (theorem 4.9 below) will be that no class $\mathrm{K}$ with $\mathrm{RRA} \subseteq \mathrm{K} \subseteq \mathcal{C}(\mathrm{RRA})$ is finitely axiomatisable.

REMARK 4.2 (for those interested in canonicity) By a result of Gehrke et al. [5, theorem 3.6], any universal class of monotone lattice expansions that is closed under completions (of a given type) is canonical — closed under canonical extensions. Since $\mathcal{C}(\mathrm{RRA})$ is such a class, $\mathcal{C}(\mathrm{RRA})$ is canonical.

Furthermore, $\mathcal{C}(\mathrm{RRA})$ is elementarily generated. This follows from Goldblatt $[8$, theorem 5.8]. Alternatively, Goldblatt showed in [9, corollary 3.4] that a canonical singletonpersistent variety is elementarily generated, and since $\mathcal{C}(\mathrm{RRA})$ is closed under completions, by $[9$, theorem 5.1$]$ it is singleton-persistent.

For simplicity, let us take an equation of the signature of relation algebras to be canonical if whenever it is valid in a relation algebra, it is valid in its canonical extension. That is to say, we restrict attention to RA. Each equation $\varepsilon$ that is preserved by completions of relation algebras is canonical - apply [5, theorem 3.6] to the class $\{\mathcal{A} \in \operatorname{RA}: \mathcal{A} \models \varepsilon\}$. So $\mathcal{C}(\mathrm{RRA})$ has a canonical equational axiomatisation.

Now by [16], RRA is barely canonical: it is a canonical variety, but every first-order axiomatisation of it has infinitely many non-canonical formulas. $\mathcal{C}(\mathrm{RRA})$, on the other hand, is a possibly rare example of a variety of relation algebras that is non-finitely axiomatisable (as we will see in theorem 4.9) and canonical, but not barely canonical, because as we just saw, it has a canonical axiomatisation. So whilst we do need infinitely many non-canonical formulas to define RRA, it seems that we can still get a long way inside RA using only canonical ones. See [11] for work along similar lines.

\subsection{Diagrams}

To prove our first main result, we will use model-theoretic diagrams. Let $\mathcal{A}=(A,+,-, 0,1$, $\left.1^{\prime},{ }^{\prime}, ;\right)$ be a finite relation algebra. The (basic) diagram $\Delta \mathcal{A}(\bar{v})$ of $\mathcal{A}$ is the conjunction of the following formulas of the signature of relation algebras, involving variables $v_{a}(a \in A)$ : $v_{a} \neq v_{b}$ for each distinct $a, b \in A, v_{0}=0, v_{1}=1, v_{1},=1, v_{-a}=-v_{a}$ and $v_{\breve{a}}=\left(v_{a}\right)^{\smile}$ for each $a \in A$, and $v_{a+b}=v_{a}+v_{b}$ and $v_{a ; b}=v_{a} ; v_{b}$ for each $a, b \in A$. Given any relation algebra $\mathcal{B}$, a map $f: \mathcal{A} \rightarrow \mathcal{B}$ is a relation algebra embedding iff $\mathcal{B} \models \Delta \mathcal{A}(\langle f(a): a \in A\rangle)$. So

$$
\mathcal{B} \models \exists \bar{v} \Delta \mathcal{A}(\bar{v}) \Longleftrightarrow \mathcal{A} \text { embeds into } \mathcal{B} \text {. }
$$

We will use diagrams in conjunction with a well-known fact about relation algebras (it holds for all discriminator varieties): for every quantifier-free formula $\varphi(\bar{x})$ of the signature of relation algebras, there is a relation algebra term $\tau_{\varphi}(\bar{x})$ such that $\varphi(\bar{x})$ is equivalent to $\tau_{\varphi}(\bar{x})=0$ in every simple relation algebra. See, e.g., [23, theorem 381]. 
DEFINITION 4.3 For a finite relation algebra $\mathcal{A}$, let $\varepsilon_{\mathcal{A}}$ be the equation $\tau_{\neg \Delta \mathcal{A}}(\bar{v})=0$.

LEMMA 4.4 For every simple relation algebra $\mathcal{B}$, we have $\mathcal{B}=\varepsilon_{\mathcal{A}}$ iff $\mathcal{A}$ does not embed into $\mathcal{B}$.

Proof. By definition, $\mathcal{B} \models \varepsilon_{\mathcal{A}}$ iff $\mathcal{B} \models \forall \bar{v}\left(\tau_{\neg \Delta \mathcal{A}}(\bar{v})=0\right)$. As $\mathcal{B}$ is simple, this is iff $\mathcal{B} \models \forall \bar{v} \neg \Delta \mathcal{A}(\bar{v})$. By (12), this is iff $\mathcal{A}$ does not embed into $\mathcal{B}$.

Now let $\mathcal{Q}$ be any non-representable simple persistently finite relation algebra.

LEMMA $4.5 \varepsilon_{\mathcal{Q}}$ is not valid in $\mathcal{Q}$.

Proof. $\quad \mathcal{Q}$ is simple and $\mathcal{Q} \subseteq \mathcal{Q}$, so $\mathcal{Q} \forall \varepsilon_{\mathcal{Q}}$ by lemma 4.4 .

LEMMA $4.6 \varepsilon_{\mathcal{Q}}$ is valid in RRA.

Proof. Let $\mathcal{A}$ be a simple representable relation algebra. Since $\mathcal{Q}$ is not representable and RRA is closed under subalgebras, $\mathcal{Q}$ does not embed into $\mathcal{A}$. By lemma $4.4, \mathcal{A} \models \varepsilon_{\mathcal{Q}}$. Since RRA is generated by its simple algebras, we deduce that RRA $\models \varepsilon_{\mathcal{Q}}$.

LEMMA $4.7 \quad \varepsilon_{\mathcal{Q}}$ is preserved by completions of relation algebras.

Proof. Let $\mathrm{Q}$ be the variety of relation algebras that validate the equation $\varepsilon_{\mathcal{Q}}$. We need to show that $\mathrm{Q}^{c} \subseteq \mathrm{Q}$.

Suppose for contradiction that there is some $\mathcal{A} \in \mathrm{Q}^{c} \backslash \mathrm{Q}$. We start by showing that $\mathcal{Q} \in \operatorname{Var}\left(\mathrm{Q}^{c}\right)$. As $\mathcal{A}$ is clearly a relation algebra, it is a subdirect product of simple relation algebras $\mathcal{S}_{i}(i \in I)$. That is, $\mathcal{A} \subseteq \prod_{i \in I} \mathcal{S}_{i}$ up to isomorphism, and the projection of $\mathcal{A}$ onto each $\mathcal{S}_{i}$ is surjective. Each $\mathcal{S}_{i}$ is therefore a homomorphic image of $\mathcal{A}$, and so $\mathcal{S}_{i} \in \operatorname{Var}\left(\mathrm{Q}^{c}\right)$ since $\mathcal{A} \in \mathrm{Q}^{c}$. If all $\mathcal{S}_{i}$ validated $\varepsilon_{\mathcal{Q}}$, then so would $\mathcal{A}$, contradicting $\mathcal{A} \notin \mathrm{Q}$. So some $\mathcal{S}_{i}$ does not validate $\varepsilon_{\mathcal{Q}}$, and as $\mathcal{S}_{i}$ is simple, we obtain $\mathcal{Q} \subseteq \mathcal{S}_{i}$ (up to isomorphism) by lemma 4.4. Thus, indeed, $\mathcal{Q} \in \operatorname{Var}\left(\mathrm{Q}^{c}\right)$.

We now cite a special case of [2, theorem 2.2], by taking ' $\mathrm{V}$ ' in that theorem to be RA:

Assume that $\mathfrak{A}$ is simple and persistently finite in RA. Then $\mathfrak{A} \in \operatorname{Var}\left(\mathrm{K}^{d} \cap \mathrm{RA}\right)$ implies that $\mathfrak{A} \in \operatorname{Var} K$ for any subclass $\mathrm{K}$ of RA.

Here, $\mathrm{K}^{d}$ denotes the class of dense extensions of members of $\mathrm{K}$. Take $\mathrm{K}=\mathrm{Q}$, observe that $\mathrm{Q}^{c} \subseteq \mathrm{Q}^{d} \cap \mathrm{RA}$, so that $\mathcal{Q} \in \operatorname{Var}\left(\mathrm{Q}^{d} \cap \mathrm{RA}\right)$, and deduce from the theorem that $\mathcal{Q} \in \operatorname{Var} \mathrm{Q}=\mathrm{Q}$. So $\mathcal{Q} \models \varepsilon_{\mathcal{Q}}$, contradicting lemma 4.5 .

COROLLARY 4.8 There are no non-representable simple persistently finite relation algebras in $\mathcal{C}(\mathrm{RRA})$.

Proof. It is immediate from lemmas $4.6-4.7$ and definition 4.1 of $\mathcal{C}(\mathrm{RRA})$ that $\varepsilon_{\mathcal{Q}}$ is valid in $\mathcal{C}(\mathrm{RRA})$. But by lemma 4.5 , it is not valid in $\mathcal{Q}$. Since $\mathcal{Q}$ was arbitrary, we are done. 


\subsection{Non-finite axiomatisability}

We now prove our first main result. The previous meaning of $n(n=5)$ is no longer needed.

THEOREM 4.9 No class $\mathrm{K}$ with $\mathrm{RRA} \subseteq \mathrm{K} \subseteq \mathcal{C}(\mathrm{RRA})$ is finitely axiomatisable (i.e., definable by a single first-order sentence).

Proof. For each $d \geq 1$, the algebra $\mathcal{A}^{(d)}$ is a simple persistently finite non-representable relation algebra (lemmas 3.17 and 3.19, and corollaries 3.16 and 3.14). So by corollary 4.8, $\mathcal{A}^{(d)} \notin \mathcal{C}(\mathrm{RRA})$, and hence $\mathcal{A}^{(d)} \notin \mathrm{K}$.

Now let $\mathcal{B}$ be a nonprincipal ultraproduct of the $\mathcal{A}^{(d)}$ for $d \geq 1$. Let $n \geq 4$. Since $\mathrm{RA}_{n}$ is a variety (fact 3.12(4)), it is elementary, and by Łoś's theorem, elementary classes are closed under ultraproducts. By corollary 3.14 and fact $3.12(1), \mathcal{A}^{(d)} \in \mathrm{RA}_{d+3} \subseteq \mathrm{RA}_{n}$ for all $d \geq n$. It follows that $\mathcal{B} \in \mathrm{RA}_{n}$.

This holds for all $n$, so $\mathcal{B} \in \bigcap_{n \geq 4} \mathrm{RA}_{n}=\mathrm{RRA}$ (fact 3.12(3)). The theorem now follows by Łoś's theorem in the usual way: if $\mathrm{K}$ is finitely axiomatisable then so is its complement, which is therefore elementary and closed under ultraproducts, so it cannot be that $\mathcal{A}^{(d)} \notin \mathrm{K}$ for all $d$, and yet an ultraproduct of the $\mathcal{A}^{(d)}$ is in RRA and hence in K.

As shown in the proof above, for each $n \geq 4$ we have $\mathcal{A}^{(n)} \in \mathrm{RA}_{n} \backslash \mathcal{C}(\mathrm{RRA})$, and hence $\mathrm{RA}_{n} \nsubseteq \mathcal{C}(\mathrm{RRA})$ and $\mathcal{C}(\mathrm{RRA}) \subsetneq \mathrm{RA}$. For those interested, because $\mathcal{A}^{(d)} \in \mathfrak{R a C A} \mathrm{A}_{d+3}$ (see the comments after corollary 3.14), we also have $\mathfrak{R a C A} A_{n} \nsubseteq \mathcal{C}(\mathrm{RRA})$ for every finite $n$. In some sense, $\mathcal{C}(\mathrm{RRA})$ is going off in a different direction from these classes.

Theorem 4.9 still holds if we replace $\mathcal{C}(\mathrm{RRA})$ by the variety NSPF defined by $\left\{\varepsilon_{\mathcal{Q}}: \mathcal{Q}\right.$ a non-representable simple persistently finite relation algebra\}, or even the variety $A D$ defined by $\left\{\varepsilon_{\mathcal{A}^{(d)}}: d \geq 1\right\}$, plus in both cases the equations defining relation algebras. The proof is the same. Since $\mathcal{C}(R R A) \subseteq N S P F \subseteq A D$, these are formally more general results, but the definitions of NSPF and $A D$ are perhaps less attractive than that of $\mathcal{C}(R R A)$.

\subsection{Maddux's variety is not finitely axiomatisable}

In [24], Maddux defined the variety $V$ generated by the completions of representable relation algebras. In symbols, $V=\operatorname{Var}\left(\operatorname{RRA}^{c}\right)$. We discussed it in the introduction, and we can now prove our second main result, solving Maddux's problem 1.1(2) from [24].

THEOREM 4.10 $V$ is not finitely axiomatisable.

Proof. As is easily seen, RRA $\subseteq V \subseteq \mathcal{C}(\mathrm{RRA})$, so the result follows from theorem 4.9.

\section{Conclusion}

We have constructed non-representable simple persistently finite measurable relation algebras $\mathcal{A}^{(d)}$, for finite $d \geq 1$, that are 'arbitrarily representable', and in fact, $\mathcal{A}^{(d)} \in$ 
$\mathrm{RA}_{d+3} \backslash \mathrm{RA}_{d+4}$. We used them to show that various varieties of relation algebras are not finitely axiomatisable, including Maddux's variety $V=\operatorname{Var}\left(\operatorname{RRA}^{c}\right)$ - this solves [24, problem 1.1(2)] negatively.

We end with some comments.

When $d=1$, the construction that we have given is a special case of the one in [3], which essentially replaces our field $\mathcal{F}$ by any finite abelian group $\mathfrak{F}$. The machinery of coset relation algebras is used in [3], and our construction (for all $d \geq 1$ ) can be shown directly to fit into that framework - for example, the required automorphisms can be shown to exist. [3] constructed a relation algebra from the cube $\mathfrak{F}^{3}$ of $\mathfrak{F}$. We too could have worked with $V_{3}^{(d)}$, which for $d=1$ is isomorphic to $\mathcal{F}^{3}$, but using $V_{5}^{(d)}$ seems to allow a simpler presentation. For example, we did not need automorphisms.

We did not use $V_{n}^{(d)}$ for $n>5$ here, but perhaps they might be useful for other things.

We assumed that our field $\mathcal{F}$ has characteristic 2. This assumption was not used often, and sometimes, such as in the proof of lemma 2.11, the use was only cosmetic. It is unclear whether the assumption can be relaxed, and what is to be gained by doing so, but some parts of the argument, such as the proof of lemma 2.8, do not seem straightforward to replicate in other characteristics.

We also assumed that $\mathcal{F}$ is finite. This ensured that each relation algebra $\mathcal{A}^{(d)}$ is finite, hence not in $\mathrm{RA}_{d+4}$, and persistently finite, which was needed in the proof of the first main theorem (4.9).

A relation algebra is completely representable if it has a complete representation - an embedding of it into a product of relation algebras of the form $\mathfrak{R e}(U)$ that preserves all existing sums. Givant and Andréka [6, problem 7.11] asked whether there is an atomic, complete, and representable measurable relation algebra that is not completely representable. Could the completion $\mathcal{B}^{c}$ of a nonprincipal ultraproduct $\mathcal{B}$ of the algebras $\mathcal{A}^{(d)}$ be an example? Unfortunately, assuming the continuum hypothesis, it appears that $\mathcal{B}^{c}$ is completely representable. So this line of attack does not look promising.

Finally, we list some open questions, for specialists.

PROBLEM 5.1 Here, $V$ denotes Maddux's variety $\operatorname{Var}\left(\mathrm{RRA}^{c}\right)$, and $\mathcal{C}(\mathrm{RRA})$ is as defined in definition 4.1 .

1. Do we have $V=\mathcal{C}(\mathrm{RRA})$ ?

2. Is $V$ elementarily generated? The question is motivated by the fact that $V$ is canonical. This is a simple consequence of [5, theorem 3.8], and is recorded in [15], so answering Maddux's [24, problem 1.1(3)] quoted in the introduction. All elementarily generated varieties of boolean algebras with operators are canonical [7, theorem 3.6.7], but not conversely [10].

3. We saw in remark 4.2 that $\mathcal{C}(\mathrm{RRA})$ is elementarily generated. Is there a 'nice' elementary class that generates it - for example, a finitely axiomatisable class?

4. Does $V$ have a canonical axiomatisation?

Australasian Journal of Logic (17:2) 2020, Article no. 1 
5. Do $V$ or $\mathcal{C}($ RRA) have Sahlqvist axiomatisations (cf. [11])?

6. Are the relation algebras $\mathcal{A}^{(d)}$ weakly representable (as defined in [17])? Most likely not, since [2, p.8] states that the algebras from [3], on which the $\mathcal{A}^{(d)}$ are based, are not weakly representable.

Problems 4-6 of [24, problem 1.1], quoted in the introduction, also remain open as far as we know. A positive answer to no. 4 would give a positive answer to problem 5.1(2) above, using $[8,9]$.

In case the reader is wondering, we mention that RRA is not finitely axiomatisable over $V[15$, theorem 2.2].

I dedicate this paper to Rob Goldblatt. Perhaps he may find some of it interesting, since he has worked on completions (e.g., [9]) and algebras of relations (e.g., [1]), he is familiar with coset relation algebras, and he is an authority on canonicity, which is involved in this article, though rather glancingly. The collaboration I have had with him, leading to nine joint papers from 2003 to the present day, is one of the two most fruitful of my life, and we have spent some wonderful times together. His generosity, wisdom, and magnificent New Zealand sense of humour are inspiring. I wish him the very happiest and most rewarding retirement.

\section{References}

[1] H. Andréka, R. Goldblatt, and I. Németi, Relativised quantification: Some canonical varieties of sequence-set algebras, J. Symbolic Logic 63 (1998), 163-184.

[2] H. Andréka and I. Németi, Varieties generated by completions, Algebra Universalis 80 (2019), 30, https://doi .org/10.1007/s00012-019-0602-8.

[3] H. Andréka, I. Németi, and S. Givant, Nonrepresentable relation algebras from groups, Rev. Symbolic Logic (2019), https://doi.org/10.1017/S1755020319000224.

[4] M. Frias and R. D. Maddux, Non-embeddable simple relation algebras, Algebra Universalis 38 (1997), 115-135.

[5] M. Gehrke, J. Harding, and Y. Venema, MacNeille completions and canonical extensions, Trans. Amer. Math. Soc. 358 (2006), 573-590.

[6] S. Givant and H. Andréka, A representation theorem for measurable relation algebras, Ann. Pure. Appl. Logic 169 (2018), 1117-1189.

[7] R. Goldblatt, Varieties of complex algebras, Ann. Pure. Appl. Logic 44 (1989), 173242.

[8] _ Elementary generation and canonicity for varieties of boolean algebras with operators, Algebra Universalis 34 (1995), 551-607.

Australasian Journal of Logic (17:2) 2020, Article no. 1 
[9] _ Persistence and atomic generation for varieties of boolean algebras with operators, Studia Logica 68 (2001), no. 2, 155-171.

[10] R. Goldblatt, I. Hodkinson, and Y. Venema, Erdős graphs resolve Fine's canonicity problem, Bull. Symbolic Logic 10 (2004), 186-208.

[11] C. Hampson, S. Kikot, A. Kurucz, and S. Marcelino, Non-finitely axiomatisable modal product logics with infinite canonical axiomatisations, Ann. Pure. Appl. Logic (2020), https://doi.org/10.1016/j.apal.2020.102786.

[12] R. Hirsch and I. Hodkinson, Relation algebras from cylindric algebras, II, Ann. Pure. Appl. Logic 112 (2001), 267-297.

[13] _ Relation algebras by games, Studies in Logic and the Foundations of Mathematics, vol. 147, North-Holland, Amsterdam, 2002.

[14] I. Hodkinson, Atom structures of cylindric algebras and relation algebras, Annals of Pure and Applied Logic 89 (1997), 117-148.

[15] - On the variety generated by completions of representable relation algebras, Algebra Universalis 81 (2020), 10. https ://doi .org/10.1007/s00012-020-0643-z.

[16] I. Hodkinson and Y. Venema, Canonical varieties with no canonical axiomatisation, Trans. Amer. Math. Soc. 357 (2005), 4579-4605.

[17] B. Jónsson, Representation of modular lattices and of relation algebras, Trans. Amer. Math. Soc. 92 (1959), 449-464.

[18] _ _ Varieties of relation algebras, Algebra Universalis 15 (1982), 273-298.

[19] R. D. Maddux, Some varieties containing relation algebras, Trans. Amer. Math. Soc. 272 (1982), no. 2, 501-526.

[20] _ A sequent calculus for relation algebras, Annals of Pure and Applied Logic 25 (1983), 73-101.

[21] _ Nonfinite axiomatizability result for cylindric and relation algebras, Journal of Symbolic Logic 54 (1989), no. 3, 951-974.

[22] - Introductory course on relation algebras, finite-dimensional cylindric algebras, and their interconnections, Algebraic logic (Amsterdam) (H. Andréka, J. D. Monk, and I. Németi, eds.), Colloq. Math. Soc. J. Bolyai, vol. 54, North-Holland, 1991, pp. 361-392.

[23] _ Relation algebras, Studies in Logic and the Foundations of Mathematics, vol. 150, Elsevier, Amsterdam, 2006.

Australasian Journal of Logic (17:2) 2020, Article no. 1 
[24] _ Subcompletions of representable relation algebras, Algebra Universalis 79 (2018), 20, https://doi.org/10.1007/s00012-018-0493-0.

[25] J. D. Monk, On representable relation algebras, Michigan Mathematics Journal 11 (1964), 207-210.

[26] Completions of Boolean algebras with operators, Mathematische Nachrichten 46 (1970), 47-55.

[27] A. Tarski, Contributions to the theory of models, III, Koninkl. Nederl. Akad. Wetensch Proc. 58 (= Indag. Math. 17) (1955), 56-64.

Australasian Journal of Logic (17:2) 2020, Article no. 1 